\title{
Review Article \\ Disease-Associated Circular RNAs: From Biology to Computational Identification
}

\author{
Min Tang, ${ }^{1}$ Ling Kui $\mathbb{D},{ }^{2}$ Guanyi Lu, ${ }^{3}$ and Wenqiang Chen $\mathbb{D}^{4}$ \\ ${ }^{1}$ School of Life Sciences, Jiangsu University, 301 Xuefu Road, Zhenjiang, Jiangsu 212013, China \\ ${ }^{2}$ Dana-Farber Cancer Institute, Harvard Medical School, Boston, MA 02215, USA \\ ${ }^{3}$ Beijing Institute of Pharmacology and Toxicology, 27 Taiping Road, Beijing 100850, China \\ ${ }^{4}$ Section on Integrative Physiology and Metabolism, Joslin Diabetes Center, Harvard Medical School, 1 Joslin Place, Boston, \\ MA 02115, USA \\ Correspondence should be addressed to Wenqiang Chen; wenqiang.chen@joslin.harvard.edu
}

Received 3 June 2020; Accepted 10 July 2020; Published 18 August 2020

Guest Editor: Tao Huang

Copyright (c) 2020 Min Tang et al. This is an open access article distributed under the Creative Commons Attribution License, which permits unrestricted use, distribution, and reproduction in any medium, provided the original work is properly cited.

\begin{abstract}
Circular RNAs (circRNAs) are endogenous RNAs with a covalently closed continuous loop, generated through various backsplicing events of pre-mRNA. An accumulating number of studies have shown that circRNAs are potential biomarkers for major human diseases such as cancer and Alzheimer's disease. Thus, identification and prediction of human disease-associated circRNAs are of significant importance. To this end, a computational analysis-assisted strategy is indispensable to detect, verify, and quantify circRNAs for downstream applications. In this review, we briefly introduce the biology of circRNAs, including the biogenesis, characteristics, and biological functions. In addition, we outline about 30 recent bioinformatic analysis tools that are publicly available for circRNA study. Principles for applying these computational strategies and considerations will be briefly discussed. Lastly, we give a complete survey on more than 20 key computational databases that are frequently used. To our knowledge, this is the most complete and updated summary on publicly available circRNA resources. In conclusion, this review summarizes key aspects of circRNA biology and outlines key computational strategies that will facilitate the genome-wide identification and prediction of circRNAs.
\end{abstract}

\section{Introduction}

Circular RNAs (circRNAs) are traditionally viewed as noncoding RNAs that form a covalently closed continuous loop and thought to be generated from imperfect splicing. However, emerging evidence has shown a complexity of circRNAs in gene expression regulation, and thus the notion that circRNAs are of low abundance has been gradually challenged. Thus, the generation of circRNAs from such noncanonical RNA splicing appears to be a feature of human gene expression [1].

Recent evidence has shown that circRNAs can act as microRNAs (miRNAs) and protein sponges as well as regulators for translation and posttranslation (Figure 1) [2-5], although other functions are also reported $[6,7]$. Contemporaneous studies have revealed that dysfunction of circRNAs is closely linked to a broad range of diseases, including cancer [8-10], cardiovascular diseases and metabolic disorders [11-15], and neurodegenerative diseases [16-18]. Also, due to notable features such as stability, high abundance in body fluids, and high cell- and tissue-specificity, circRNAs exhibit great potential to serve as biomarkers for diseases [19-22].

In the present review, we will briefly introduce the biology of circRNAs, including the biogenesis process, classification, and characteristics. Given that the biological function and mechanisms of gene regulation of circRNAs are not fully understood, we will summarize what has been widely acknowledged. In addition, since several features of circRNAs, including circular conformation, relatively low abundance, and overlap in sequence with other RNA counterparts, often hinder the investigation of circRNAs, we will then describe recent progress in computational strategies for identification and prediction of circRNAs. In contrast to 


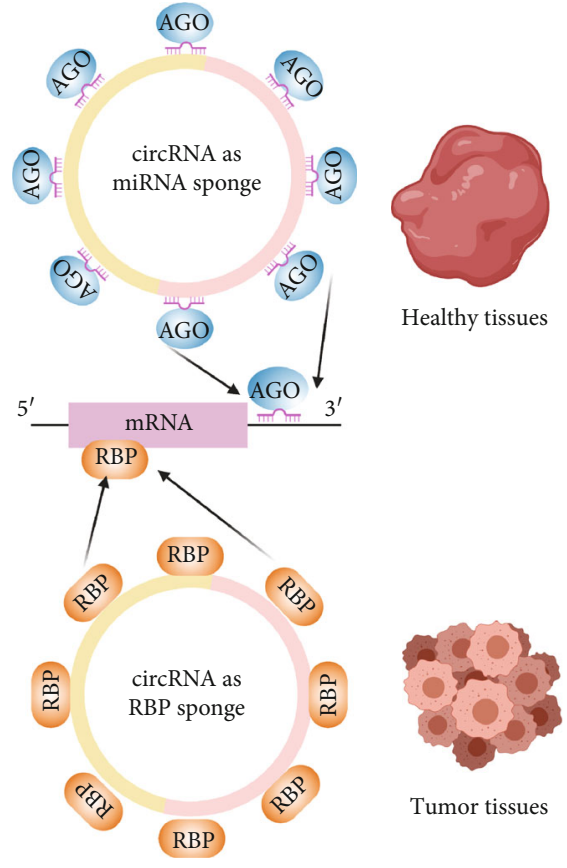

(a)

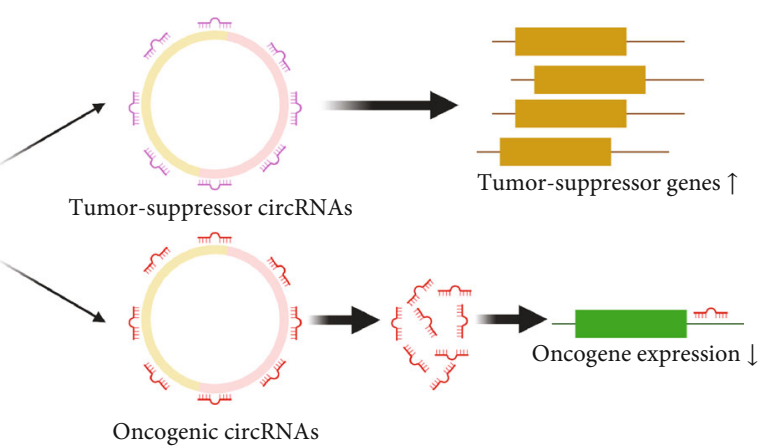

(b)

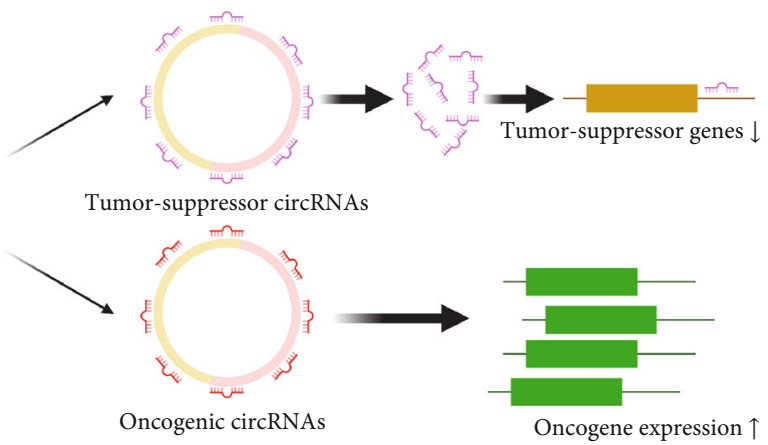

(c)

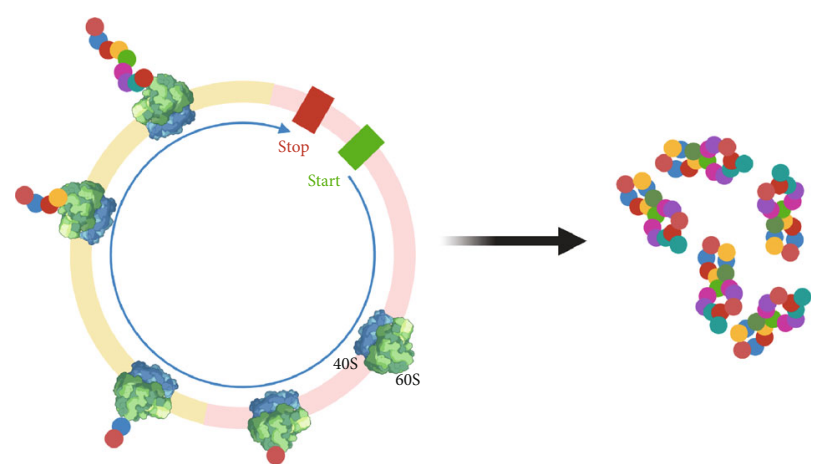

(d)

Figure 1: Translation of circRNAs. (a) circRNAs can serve as a miRNA sponge, containing multiple binding sites for miRNAs (blue) or RBPs (in red), thus affecting gene regulation. (b and c) Illustrations show the role of circRNAs as miRNAs in healthy and tumor tissues. Tumorsuppressor circRNA sponges contain binding sites for tumor-suppressor miRNAs (light purple), while oncogenic circRNA sponges contain binding sites for oncogenic miRNAs (red). Tumor-suppressor circRNAs upregulate tumor-suppressor genes (yellow) in healthy tissues but downregulate these genes in tumor tissues, whereas oncogenic circRNAs suppress oncogene (green) expression in healthy tissues but upregulate these genes in tumor tissues. AGO: Argonaute; RBP: RNA-binding protein. Illustration is inspired by and modified from [164]. (d) New studies suggest that circRNAs generated by backsplicing are able to be translated into proteins. Illustration is modified from [38, 39]. Illustrations were generated using BioRender.

benchmarking the strategies, we aim to give the readers a board introduction of circRNA biology and computational method, which will help them in designing their future studies and analyzing results. Readers interested in specific topics should refer to the reviews on circRNAs that have been summarized elsewhere [23-27].

\section{Discovery of circRNAs}

circRNAs were initially discovered via electron microscopy as a viroid in the mid-70s, because of the circular conformation $[28,29]$. The biological analysis found that these cir-
cRNAs show several features, including (1) single-stranded, (2) high thermal stability, (3) self-complementarity in a rod-like structure, and (4) covalently closed as a loop [28]. Later, in the 90s, owing to advancement in computational biology and RNA sequencing, researchers finally determined the structure of the previously identified transcripts that show an inverted order of exons that is distinct from genomic DNA, which was mistakenly recognized as RNA splicing errors [30]. This study found that, although these transcripts are nonpolyadenylated and not as abundant as in a normal transcript, they are stable molecules and expressed in the cytoplasmic part of the cells [30]. 
The breakthrough in high-throughput sequencing (HTS) technology in the 21 st century made it possible to deepen our understanding of circRNA sequences and functionality. In 2012, using deep RNA sequencing (RNA-seq) of normal and cancer stem cells from human samples, circRNAs were identified from a substantial fraction of spliced precursor message RNAs (pre-mRNAs) that showed a noncanonical order [1], suggesting a new feature of the gene expression program in human cells. Later, a close examination of circRNAs using Circle-Seq found that these molecules usually consist of up to five exons; however, each of them can be three times longer than the average expressed exon [31, 32]. A computational strategy was developed to specifically detect circRNAs, enabling identification of thousands of stable circRNAs [32]. As a proof-of-concept, using biochemical, functional, and computational analyses, this study showed that CDRlas, a known human circRNA, can bind miR-7 in neuronal tissues to function as a negative regulator [32].

Interestingly, treating RNAs with RNA exonuclease to deplete linear RNAs, researchers were able to perform bioinformatic analysis to identify complementary ALU repeats in introns; the results showed that circRNAs are abundant and stable RNA splicing products and are not randomly produced, suggesting that circRNAs are truly involved in gene expression regulation [31]. It is worth noting that all these discoveries would not have been made possible without the advancement of HTS technology.

\section{Characterization of circRNAs}

Thanks to the efforts from a number of research groups, to date, more than 20,000 different circRNAs have been identified, showing an unprecedented diversity of circRNAs among different species [33]. In addition, tissue and subcellular expression are also characterized. Surprisingly, in mammalians, most circRNAs are found in the brain, mainly in neuronal and synaptic functions $[34,35]$. In situ sequencing was used to reveal the subcellular localization of circRNAs in the brain and found that as predicted, circRNA transcripts are enriched in the cytoplasm. However, nuclear localization was also found, though to a less extent [36]. Other studies also showed the role of circRNAs to regulate gene expression in the nucleus [4]. In other tissue types, such as the liver, heart, placenta, and blood, circRNAs are also found [36]. Another study not only investigated tissuespecific expression pattern but also explored the role of circRNAs in a development stage-specific manner and found that similar to adult human tissues, fetal tissues show an abundance of circRNAs [37].

Before we discuss the classifications of circRNAs, we will briefly introduce the noncoding RNA (ncRNA) family. As its name suggests, ncRNA is an RNA that is not translated into a protein. ncRNAs mainly consist of transfer RNA, ribosomal RNA (rRNA), and many other small RNAs such as long noncoding RNA (lncRNA: $\geq 200 \mathrm{nt}$ ), small noncoding RNA (sncRNA: 100-200 nt), miRNA (20-24 nt), and endogenous small interfering RNA (endo-siRNA). circRNAs have been categorized as ncRNAs; however, recent new studies challenged this view by demonstrating that circRNAs can code for proteins (Figure 1(d)) [38-40]. These studies showed that a group of circRNAs termed ribo-circRNAs, because they are associated with translating ribosomes, are bound by membrane-associated ribosomes, suggesting the existence of unexplored modes of regulation of genes and proteins $[38,39]$. Another study showed that translation of circRNAs could be driven by $\mathrm{m} 6 \mathrm{~A}$, the most abundant RNA modification [41]. Nevertheless, the characterizations of circRNAs have just started.

Stability is one of the distinct characteristics of circRNAs separating them from linear RNAs. In general, compared to linear RNAs, circRNAs are quite stable, because the lack of a poly(A) tail in circRNAs can protect them from exonuclease-mediated degradation [31]. This feature has been utilized to a recent engineering study to generate exogenous circRNAs, thus obtaining more potent and durable proteins in eukaryotic cells [42].

\section{Biogenesis of circRNAs}

Linear RNAs usually terminate with $5^{\prime}$ caps and $3^{\prime}$ tails and undergo canonical splicing; however, due to the closed loop structure, neither $5^{\prime}$-to- $3^{\prime}$ polarity nor poly(A) tail can be found in circRNAs. Thus, circRNAs show stability over linear RNAs [31,32]. Canonical splicing in pre-mRNAs is catalyzed by a spliceosome assembly, resulting in a linear RNA transcript with a $5^{\prime}$-to- $3^{\prime}$ polarity. This splicing strategy is considered as highly efficient. Different from canonical splicing, circRNAs are generated via backsplicing, which, on the contrary, is considered as a noncanonical way (Figure 2). When the upstream $3^{\prime}$ splice acceptor site joins with a downstream $5^{\prime}$ splice donor site, the junction site is ligated by a $3^{\prime}$ $-5^{\prime}$ phosphodiester bond, resulting in covalently closed circRNAs. The sizes of mature circRNAs have a wide range from $\sim 100 \mathrm{nt}$ to $4 \mathrm{~kb}$ [43]. In human cells, the most common size is several hundred nucleotides spanning two or three exons [31, 44, 45]. Besides, long flanking introns comprising inverted repeat sequences have been proved to promote exon circularization [46, 47]. Unlike canonical splicing, backsplicing is usually considered as poorly efficient by approximately $1-3 \%$ of the former $[48,49]$.

\section{Categories of circRNAs}

The RNA research community has annotated four different types of alternative splicing, including (1) intron retention, (2) exon skipping, (3) alternative $5^{\prime}$ splicing, and (4) alternative $3^{\prime}$ splicing [50], suggesting the complexity of the biogenesis of circRNAs. Based on these four types of alternative splicing, circRNAs can be categorized into four types: intron-derived circRNAs, exon-derived circRNAs (ecircRNAs), intergenic circRNAs, and exon-intron circRNAs (elciRNAs) [51]. Among these types, ecircRNAs are predominantly generated from backspliced exons as the largest type of circRNAs, accounting for the majority of the circRNAs that have been discovered. 


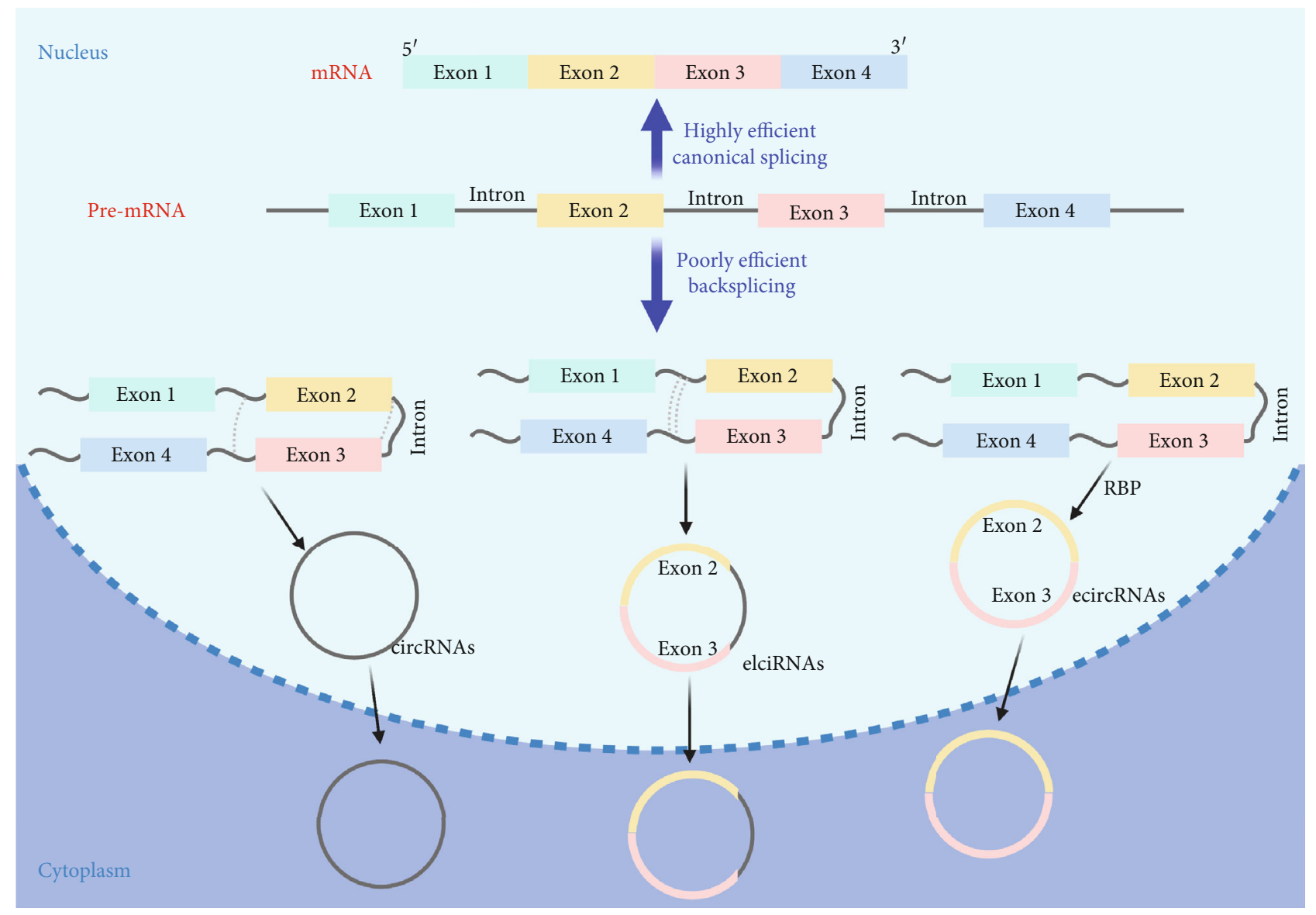

Figure 2: Biogenesis of circRNAs. Pre-mRNAs go through two splicing pathways to generate a linear RNA via highly efficient canonical splicing (top) or to produce circRNAs and an alternatively spliced linear RNA via poorly efficient backsplicing. As a result, different types of circRNAs can be produced (see discussion in text). Illustration was generated using BioRender.

\section{Major Biological Function and Disease Relevance}

In contrast to mRNAs and miRNAs, the biological functions of circRNAs are largely unclear. However, in the last decades, a number of seminar investigations have been conducted to demonstrate a wide variety of roles that circRNAs might play. Here, we briefly summarize some critical functions that circRNAs are implied to play.

CircRNAs can act as miRNA sponges, that is to say, by its name, circRNAs are reservoirs of miRNAs (Figure 1(a)). It is well known that miRNAs belong to a family of ncRNAs that regulate gene expression in a wide range of biological processes. The current view of circRNAs as a miRNA or protein sponge is that circRNAs regulate miRNA activity, thus modulating the expression of miRNA target genes [52]. As illustrated in Figures 1(b) and 1(c), in healthy and tumor tissues, specific circRNAs harbor miRNAs that target different types of genes such as tumor-suppressor genes or oncogenes, thus exhibiting various biological effects. Owing to the importance of miRNAs that bind to circRNA sponges, miRNA-based computational pipelines have been established to predict circRNA targets. We will revisit this topic in a later section of this review. In addition to regulating
miRNA, circRNAs also serve as the sponge of RNAbinding proteins (RBPs) to regulate intracellular transport (Figure 1(a)), thereby modulating gene expression of relevant RBPs of interest [53]. Readers with interests in this topic could find more details in several recent reviews [5456]. As shown in Figure 1(a), circRNAs, such as ciRS-7, also bind to Argonaute (AGO) proteins in a miR-7-dependent manner [57], which could regulate mRNA transcription and translation.

A number of circRNAs have been identified as miRNA sponges. A prominent example is ciRS-7, which serves as a miR-7 sponge $[32,57]$. ciRS-7 is highly expressed in the cytoplasm and has more than 70 miR-7 target sites [57]. It has been reported that ciRS-7 functions as both tumorsuppressor and oncogenic sponges, serving as a promising biomarker for various cancers such as colorectal cancer [58], hepatocellular carcinoma [59], esophageal squamous cell carcinoma $[60,61]$, cervical cancer [62], and pancreatic cancer [63]. Interestingly, some studies also show that ciRS7 promotes $\beta$-amyloid precursor protein (APP) and $\beta$-site APP-cleaving enzyme (BACE1) degradation [16]; thus, it might also play a role in Alzheimer's disease.

CircRNAs have been implicated in several diseases such as cancer, cardiovascular diseases, and neurodegenerative 


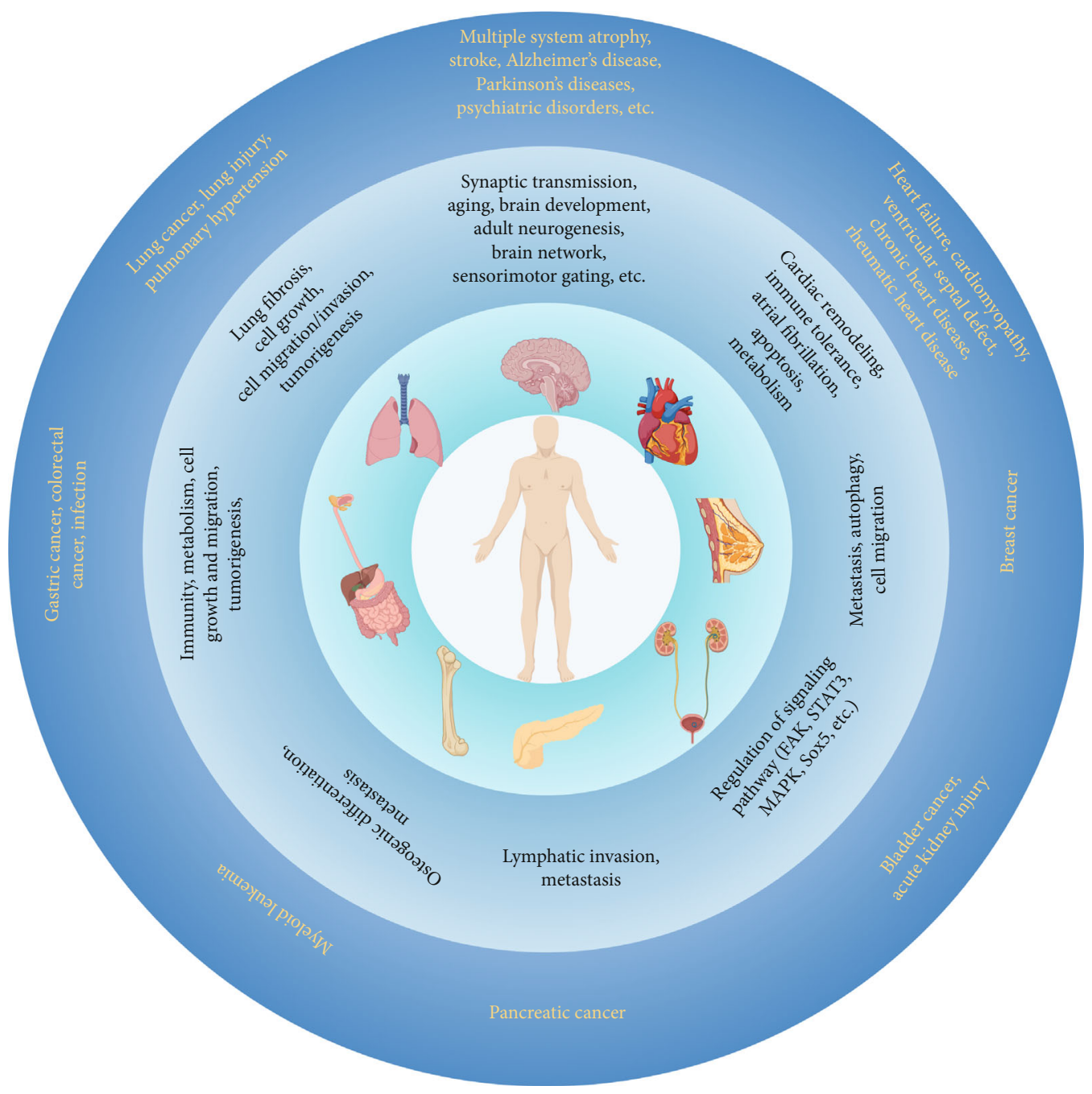

FIGURE 3: circRNAs and human diseases. circRNAs are abundantly expressed in various tissues and are implicated in a number of human diseases, including cancer and brain disorders. Illustration was generated using BioRender.

diseases. First, circRNAs are abundantly and dynamically expressed in the brain [35] and have been shown to participate in a variety of brain-related processes such as synaptic transmission [64], aging [65-67], sensorimotor gating [64], cell-type-specific interaction and brain network [68, 69], development and adult neurogenesis [70-73]. Therefore, many investigators have demonstrated that circRNAs play important roles in the pathogenesis of a number of brainrelated disorders such as multiple system atrophy [74], transient focal ischemia and stroke [75-82], neuropsychiatric disorders [64, 68], Alzheimer's disease [83, 84], Parkinson's disease [85], brain bacterial infection [86], brain tumors [87], and metabolic diseases [88]. Second, circRNAs are also highly expressed in the heart [89] and are reported to play a role in cardiac remodeling [90], stress response [91], endothelial-to-mesenchymal transition [92], metabolism [93, 94], immune tolerance [95], atrial fibrillation [96], and apoptosis [97]. Thus, these circRNAs are suggested to participate in heart diseases such as heart failure [91, 97, 98], ventricular septal defect [99], chronic heart disease [92, 100-102], alcoholic cardiomyopathy [93], and rheumatic heart disease [96]. In the lung, circRNAs have been found to be important in lung fibrosis [103], cell growth [104], cell migration and invasion [105-108], cancer tumorigenesis [109], and so on; thus, these circRNAs play a role in various lung-related diseases such as lung cancer $[105,107,110$ 112], lung injury [113], and pulmonary hypertension [114]. The role of circRNAs in various human diseases are annotated in Figure 3.

\section{Bioinformatic Analysis of circRNAs}

Given the importance of circRNAs in gene expression regulation, a growing interest emerges in identifying novel circRNAs and understanding their biological functions. Therefore, genome-wide identification and prediction of circRNAs are crucial for the study of circRNA biological functions $[115,116]$.

Effective investigation of circRNAs highlights a particular need of HST technology. In the past years, the highthroughput microarray was a dominating means to study the junction sequences of circRNAs $[117,118]$. By designing 
probes to target specific circular junction sites, a circRNA microarray allows accurate and reliable detection of individual circRNAs. Following a detailed annotation of potential miRNA target sites, a circRNA microarray helps to reveal their potential roles as a miRNA sponge. The isolated RNA samples go through a pretreatment process, in which RNase $\mathrm{R}$ is used to remove linear RNAs and improve the purity of circRNAs. However, the limited number of known circRNAs during annotation and the use of a junction sequence to identify circRNAs bring limitations to the application of a microarray. Therefore, in recent years, high-throughput RNA-seq technology has become the dominant approach to identify circRNAs. As a result, a number of computational pipelines for circRNA identification have been developed to identify circRNAs from massive RNA-seq databases.

In this section, we introduce several commonly used computational pipelines for the identification of circRNAs. Figure 4 outlines several key steps in studying circRNAs using publicly available pipelines; thus, readers could have a brief idea of where to choose individual pipelines. We apologize for omitting any key pipelines or key steps. Thus, we highly recommend readers to refer to other reviews specifically on this topic [119-122]. Table 1 provides a comprehensive summary of online tools for the study of circRNAs, while Table 2 is a list of computational pipelines for optional analysis of circRNAs. To our knowledge, this is the most comprehensive and updated summary of circRNAs tools. In addition, a video-based introduction to the identification of circRNAs from RNA-seq is also available from JOVE [123].

To effectively identify circRNAs, no matter which computational pipeline is used, one needs to discriminate circRNAs from linear RNAs. Several biochemical assays have been developed to distinguish circRNAs from other backsplicing products, including (1) divergent primer PCR, (2) relative migration of circRNAs from a canonical linear RNA in an agarose gel, (3) 2D gel electrophoresis, (4) gel trapping, and (5) exonuclease enrichment [119]. Other than biochemical enrichment strategies, deep sequencing with novel bioinformatics analysis has been developed to perform a comprehensive characterization of circRNAs. To date, candidate- or pseudo-reference-based strategies have been designed in computational pipelines $[119,124]$. The candidate-based strategy uses a list of candidate junctions that were generated from previous models $[1,119]$. Thus, this approach is able to analyze rRNA-depleted libraries in a fast manner; however, it has an obvious limitation in unannotated transcripts. Constructing putative circRNA sequences with gene annotation, a pseudo-reference-based approach, such as KNIFE [45], NCLScan [125], and PTESFinder [126], has become widely used. These approaches use several systematic filtering steps to remove false positive $[120,124]$. For example, by using PTESFinder to analyze previously mined RNA-seq reads, significantly more distinct structures were found than previously reported (between $13 \%$ and $42 \%$ ), whereas a significant number of reads were excluded by PTESFinder due to low map quality or multiple map locations [126]. Thus, owing to these novel pipelines, the highest specificity and sensitivity could be achieved. In addition to these strategies, a fragmented-based strategy is also frequently used, in which a backsplicing junction is aligned to the genome [120].

Although these detection pipelines could significantly accelerate the identification of novel circRNAs, inconsistency in results might occur when switching from one pipeline to another. Thus, evaluations for different circRNA pipelines had been performed. A recent comparison study has provided a comprehensive and unbiased comparison among several circRNA detection pipelines [120]. This study used a number of measurements to evaluate their performance, including precision, sensitivity, F1 score and area under curve, random access memory consumption, running time, and physical disk space utilization, and concluded that CIRI, CIRCexplorer, and KNIFE have better performance [120]. An earlier review had summarized the criteria in different pipelines or algorithms to perform filtering and accuracy evaluation; thus, we highly recommend readers to refer to this review [127]. In addition, it is worth noting that, as many studies have already pointed out, this study also suggested that no individual pipeline could achieve the best performance among all the metrics used, indicating an urgent need to refine and integrate all these available methods for circRNA detection $[128,129]$. For the time being, pairing different pipelines possibly produces a much more reliable output, for example, circRNA and find_circ.

To increase the accuracy in circRNA identification, two concerns should be kept in mind when designing experiments: (1) At the experimental stage, many variations can affect circRNA abundances, such as RNA purification, size selection, and RNA fragmentation followed by adaptor ligation. RNase $\mathrm{R}$ is commonly used to digest linear RNAs to enrich circRNAs for sequencing, but not all circRNAs are resistant to RNase R; conversely, a few linear RNAs can avoid RNase R digestion. (2) A small fraction of circRNAs inherently exits in common cell lines, which account for approximately $2-4 \%$ of the total mRNAs. This level is higher in platelets. Therefore, significant biases will arise when bioinformatic analysis relies on junctional reads. As a result, a high rate of false positives occurs. To this end, most pipelines apply multiple high thresholds on absolute read counts. Other pipelines employ statistical approaches to reduce the reliance on the thresholds.

The current limitations of circRNA research include limited methods available to detect and quantify circRNAs. Although RT-qPCR-based methods are low cost and highly sensitive methods that can be easily applied in many laboratories, they are not high-throughput methods that can detect and quantify circRNAs. While RNA-seq has served as the main method that has high sensitivity and high throughput, the cost can be high, and it usually requires sufficient computational power. A detailed comparison of different methods for circRNA detection and quantification can be found elsewhere [7]. The genome-wide prediction tools, as discussed here, can largely assist in the identification and characterization of circRNAs; however, it is still challenging to assess the circRNA-miRNA and circRNA-protein interactions. In most cases, the sequences of the circRNAs are not clear, which might be problematic for downstream analysis such as 

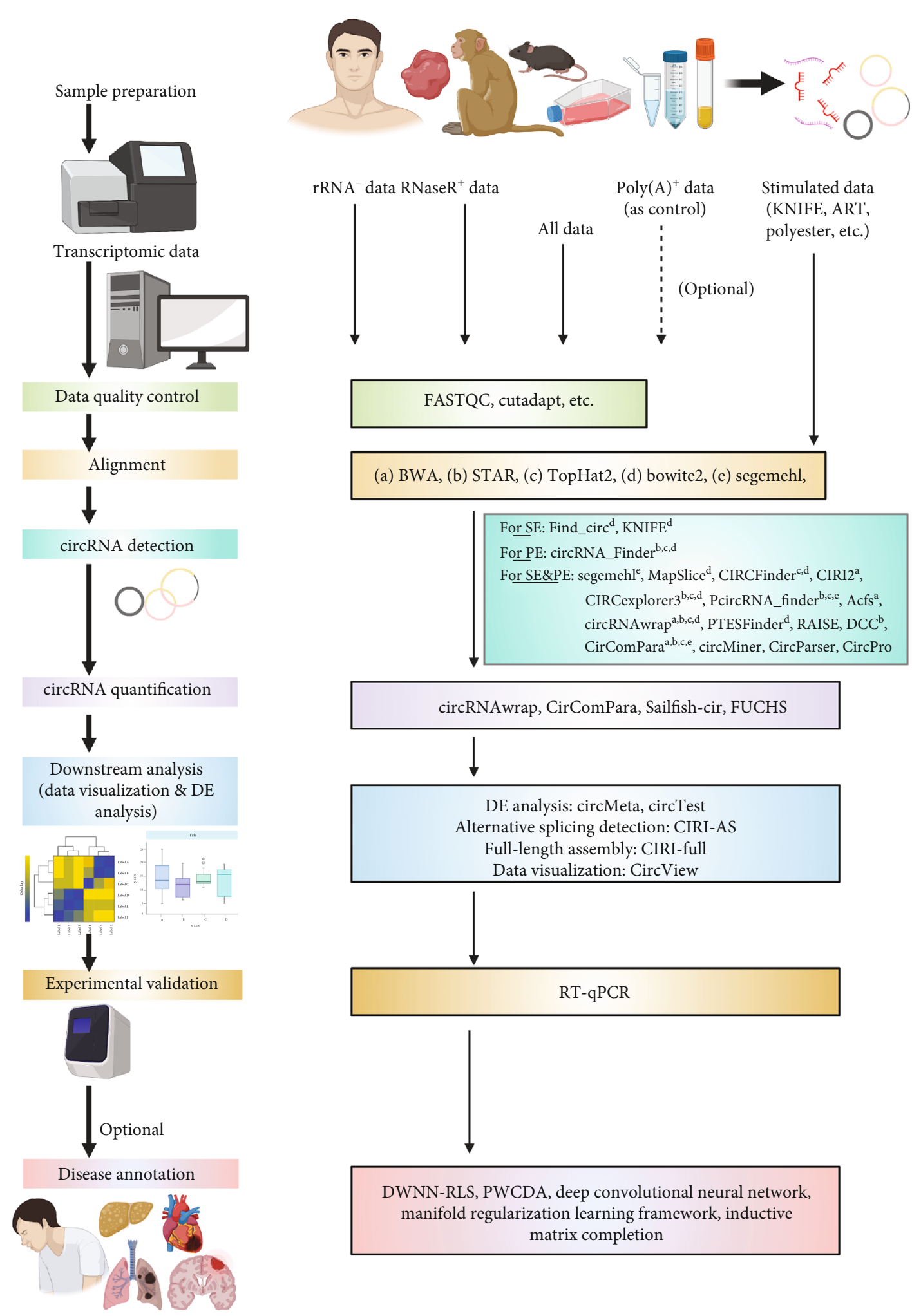

Stimulated data

(KNIFE, ART,

polyester, etc.)

(a) BWA, (b) STAR, (c) TopHat2, (d) bowite2, (e) segemehl,

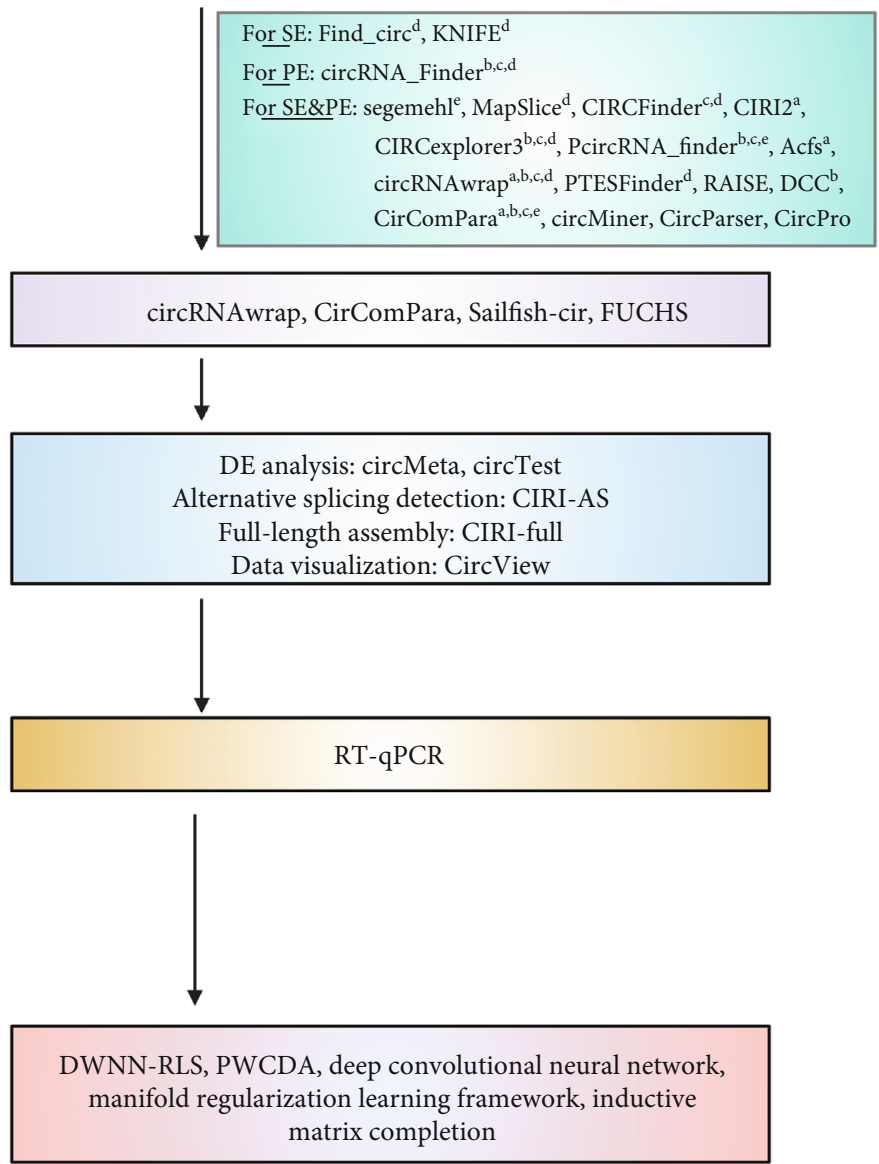

FIGURE 4: Key steps in studying circRNAs using publicly available pipelines. For read data, the library preparation is similar to traditional mRNA extraction. For stimulated data, several tools such as KNIFE and CIRI-simulator can be used. Alignment methods for linear RNAs, such as STAR and TopHat, are also commonly used for circRNAs, Therefore, a number of professional pipelines shown in Table 1 can be applied for circRNA detection, such as DCC and CIRI. For downstream analysis, other optional pipelines can be employed for different purposes. Finally, several pipelines can be used to check the association of circRNAs and diseases. The authors apologize for omitting any key pipelines or key steps. Illustration was generated using BioRender. 


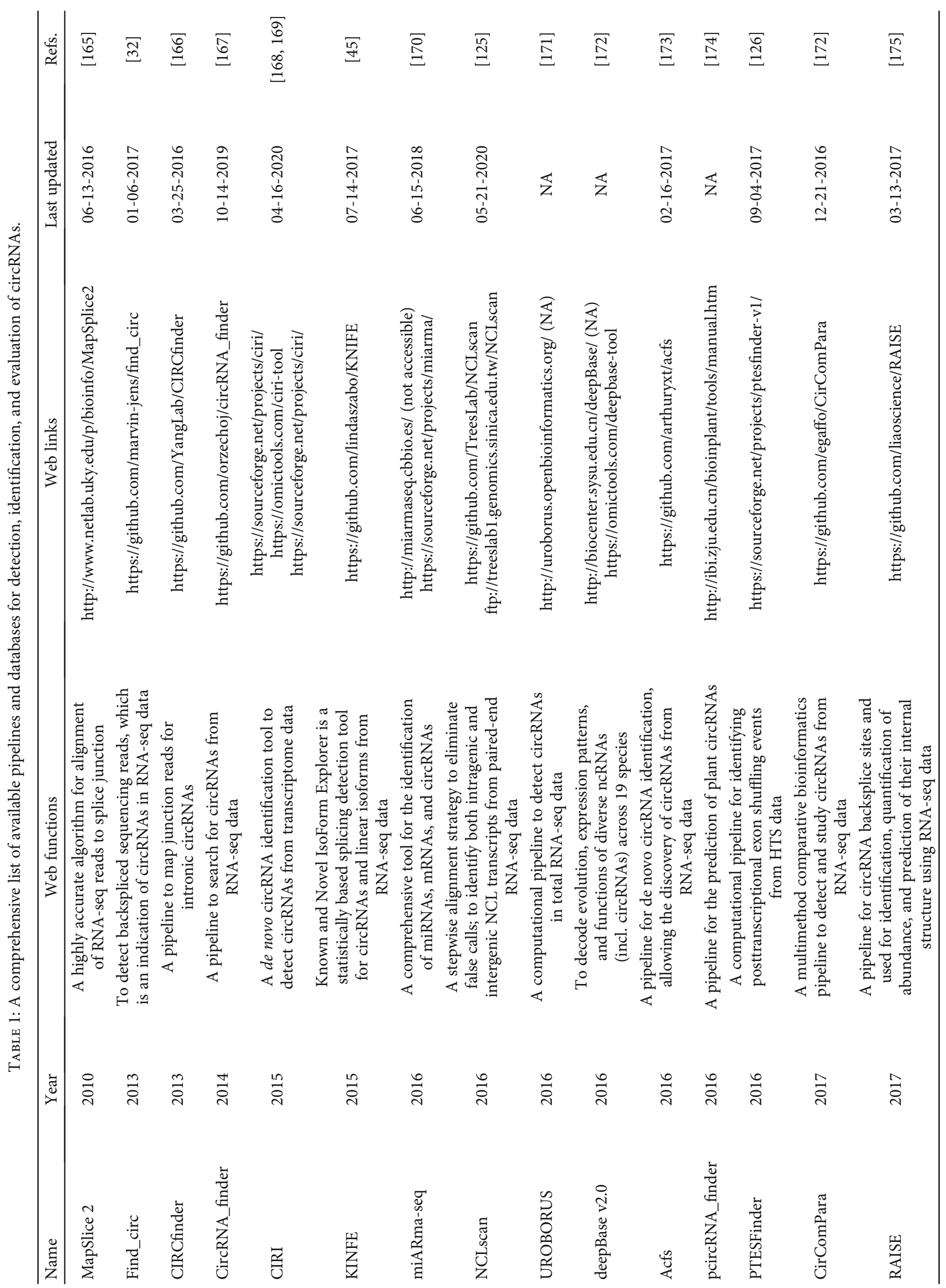




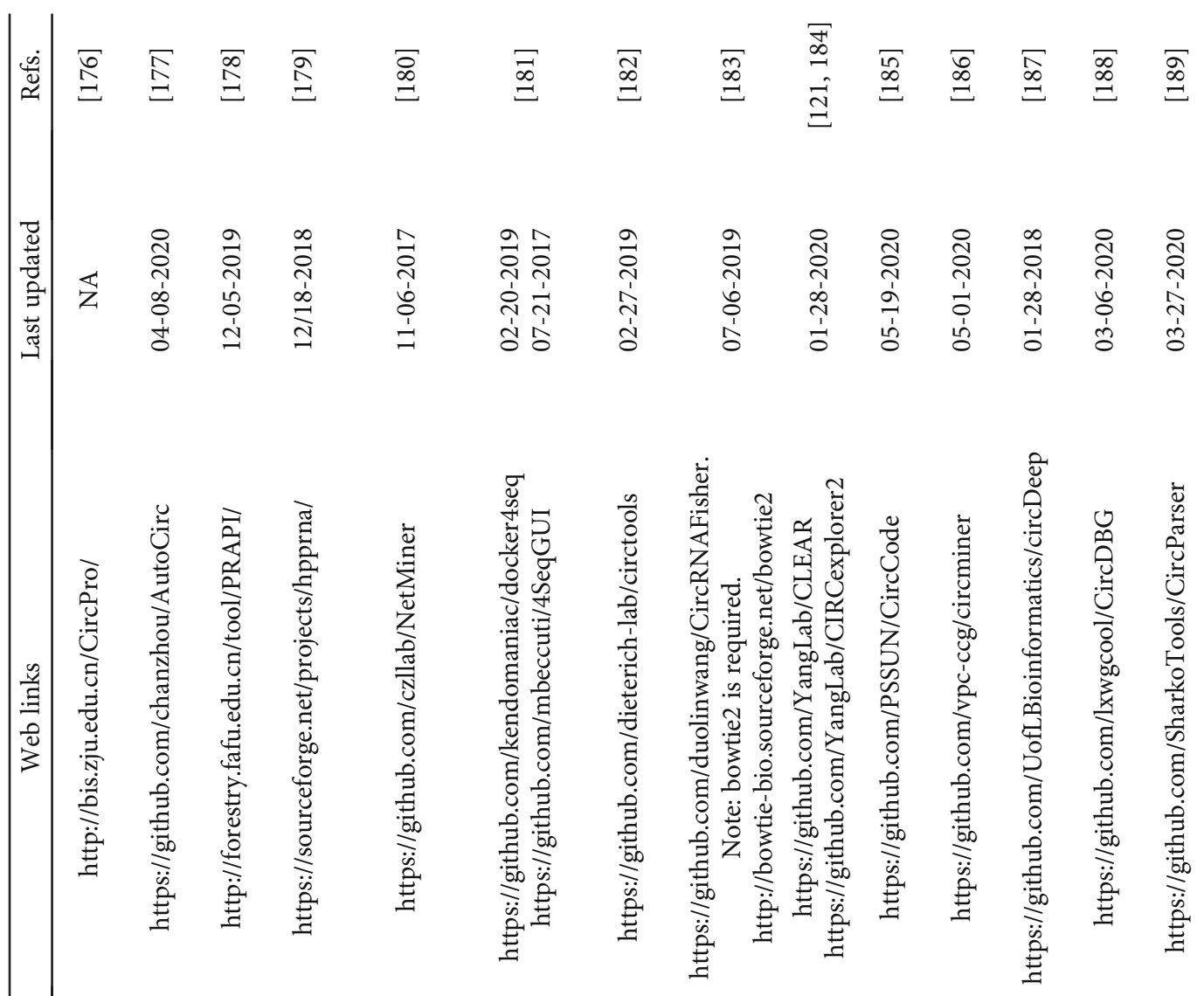

焉

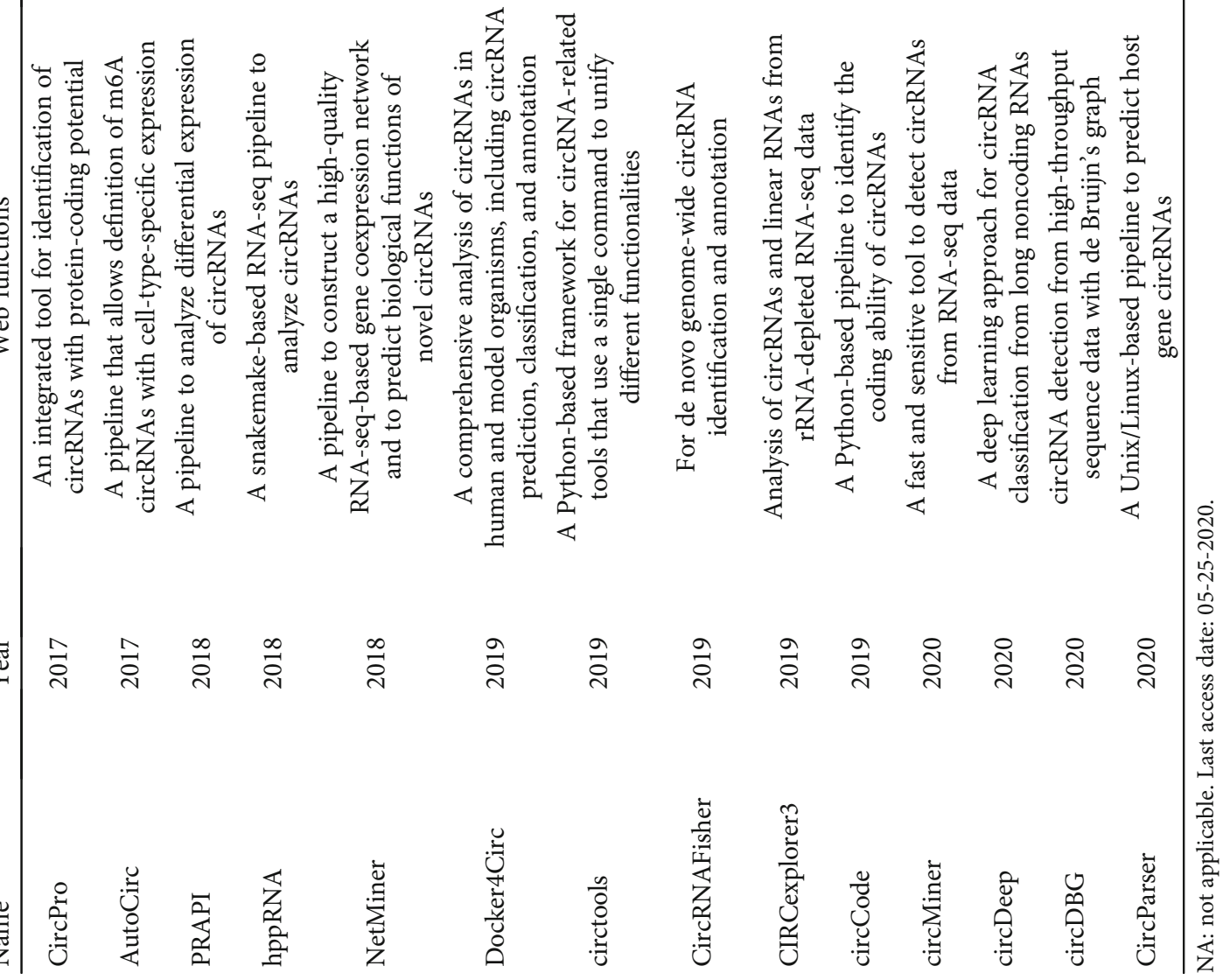




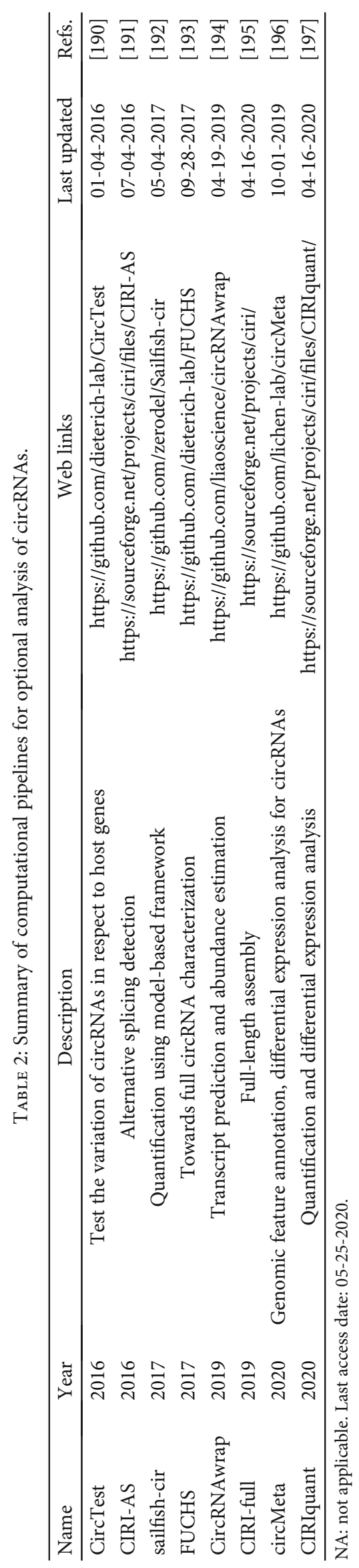


miRNA target prediction. In addition, bioinformatic analysis relying on reads spanning the backsplice junction could be problematic because of the biases in read density [127, 130]. We envision that future studies could help solve these critical issues.

In sum, although a number of pipelines are available for circRNA research, how to obtain genome-wide detection of circRNAs with high sensitivity and specificity remains a challenge. It is foreseeable that in the future, a comprehensive comparison of these pipelines, as well as a comparison in computational power using publicly available datasets, will become available.

\section{Comprehensive Databases of circRNAs}

Other than the computational tools that are used for the detection and identification of circRNAs, it is undoubtedly important that a comprehensive understanding of the association of these identified circRNAs and human diseases is eagerly expected. Therefore, several circRNA databases have been established containing thousands of mammalian circRNAs carefully selected from various sources. Thus, detailed information, such as genome sequence, subcellular location, and disease annotation, are all provided to researchers working on circRNAs.

Table 3 summarizes the most updated circRNA databases that are publicly available. Among these databases, several of them are widely used, such as Circ2Traits [131], circBase [132], and circFunBase; they are among the earliest circRNA databases that are commonly used. Here, we briefly discuss how to make full use of CircBase. We suggest that readers find more useful information from other papers $[120,132,133]$.

circBase, as one of the earliest developed databases for circRNAs, was brought in 2014 and has been widely used in the circRNA community [132]. As of today, the original report of circBase has been cited for nearly 600 times, indicating that it has been regarded as a powerful tool for the community [132]. The main aim of developing circBase was to provide summary information of individual circRNAs that have been identified, together with their genomic context. Three ways of searching circBase were provided, including simple search, list search, and table browser search. These searching methods can be easily found on the main page of the website (http://www.circbase.org/). Simple search, with identifiers, genomic coordinates, sequences, gene ontology identifiers, transcript ID, and gene symbols, is the easiest way of searching the database. List search gives users an option to paste or upload a list of several circRNAs or refseq identifiers, as well as gene symbols. Organism is required to be selected. Table browser search is a quick search option based on the browser interface. Note that organism and dataset information is required to be selected. As illustrated in Figure 5(a) as an example, in the circBase table browser page, users could select human as Organism and use a dataset from a previous study [31]. Both sample conditions and annotation allow for multiple selection. After submitting using the search button, a detailed result page will be returned, with basic information on individual circRNAs that matches the query (Figure 5(b)). The listed information includes organism data source; genomic position information which directs to a link from the UCSC genome browser, with full information on strand; circRNA ID; genomic length; spliced length; list of samples that contain the circRNAs; and number of reads. By clicking a single circRNA, the link will direct the users to a single record page, which contains detailed information on a particular circRNA. Detailed information on how to use circBase can be found on the documentation page (http://www.circbase.org/doc/help_mod.html). In addition, in circBase, data can be exported in standardized formats such as xlsx, txt, csv, or fasta, providing users a variety options to integrate with other analysis tools. In general, circBase is an excellent database that focuses on elementary information of backsplicing junction coordinates.

CircBase has been used in several key studies to identify targeted circRNAs, and this identification can be validated by quantitative real-time PCR or downstream analysis. Thus, circBase plays an important role in the identification of potential biomarkers for various cancers [134-143].

Here, we are giving the readers another example, circad (circRNAs associated with diseases), a database mainly for disease-associated circRNAs [144]. After submitting a circRNA's name in the browser (http://clingen.igib.res.in/ circad/), the database returns with a selection of different organisms. Selecting one organism will bring users to the next page, which has information including genome locus, gene name, disease association, fold change, and a publication's PubMed ID (PMID). It is worth noting that as an exception to many databases, circad includes detailed information of the primers used in that publication. A detailed documentation on how to use circad can be found (http://clingen.igib.res.in/circad/img/circad.pdf).

In addition, several other databases have also been developed. Here, we provide a brief introduction to each of them (for the web links and last updated data, as well as references for individual databases, please refer to Table 3):

(1) circ2Traits is the first comprehensive database of potential disease association of circRNAs in humans [131]; in this database, users can find SNPs associated with diseases and AGO interaction sites

(2) SomaniR is a database mainly for cancer somatic mutation in miRNAs and their target sites that might potentially interact with circRNAs [145]

(3) CircNet is a database with resources of novel circRNAs, integrated miRNA-target network, expression, annotations, and sequences of circRNA isoforms [146]

(4) $\operatorname{circRNADb}$ is a human circRNA database that contains more than $32 \mathrm{k}$ annotated exonic circRNAs [147]

(5) TSCD is an integrated tissue-specific circRNA database, which deposits features of tissue-specific circRNAs [148], and users could find tissuespecific expression in both mouse and human adult and fetus 


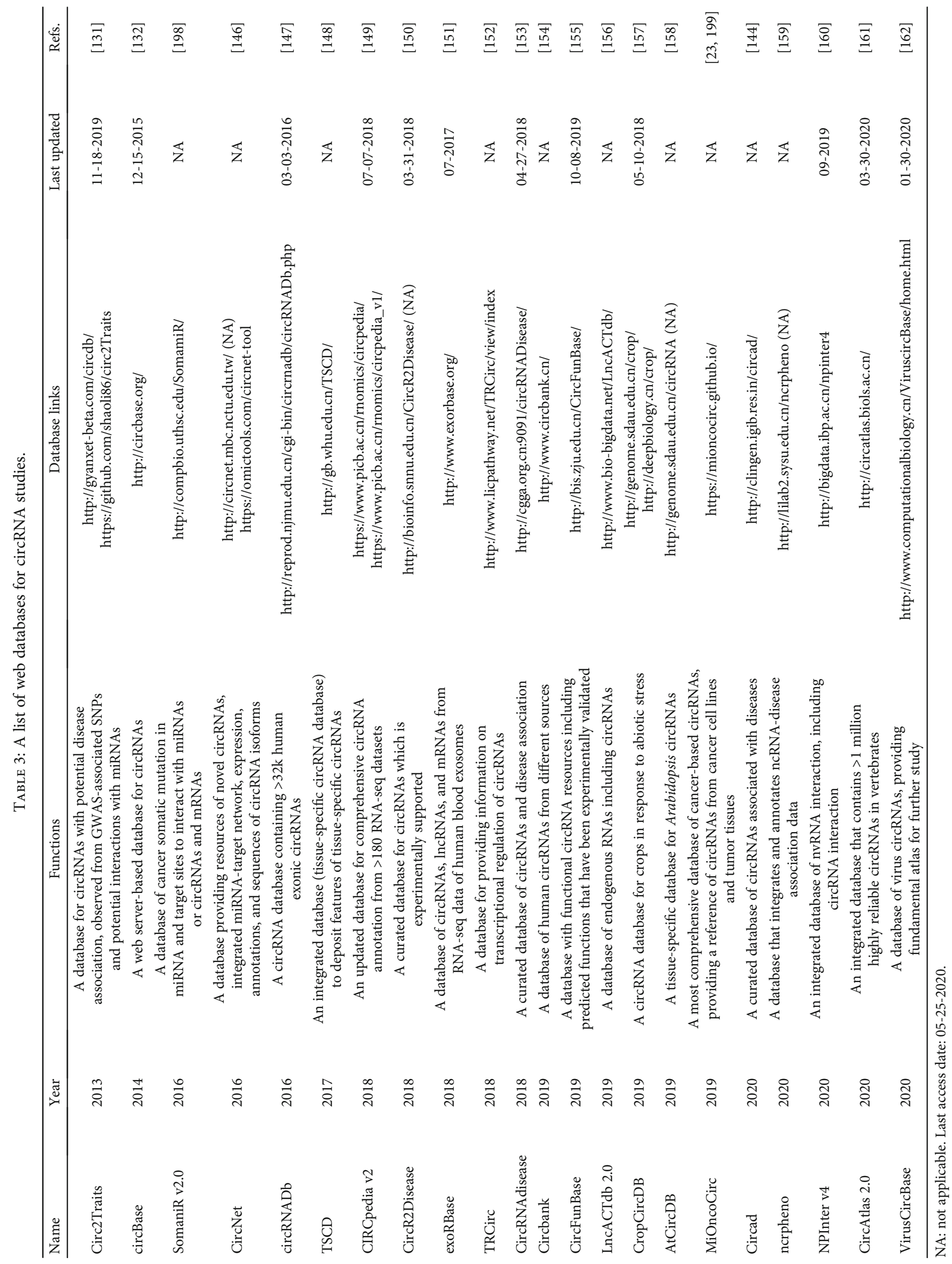




\section{circBase}

home list search table browser blat downloads help

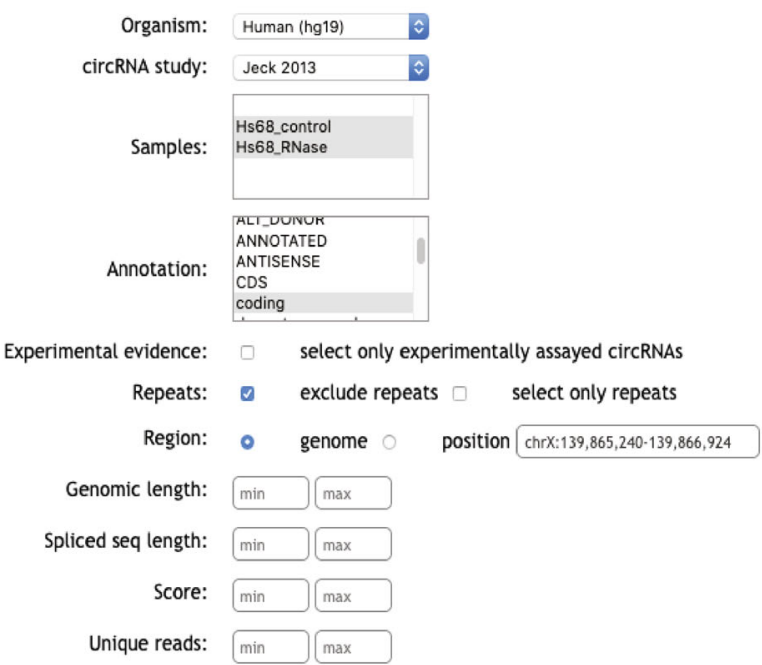

\section{circBase}

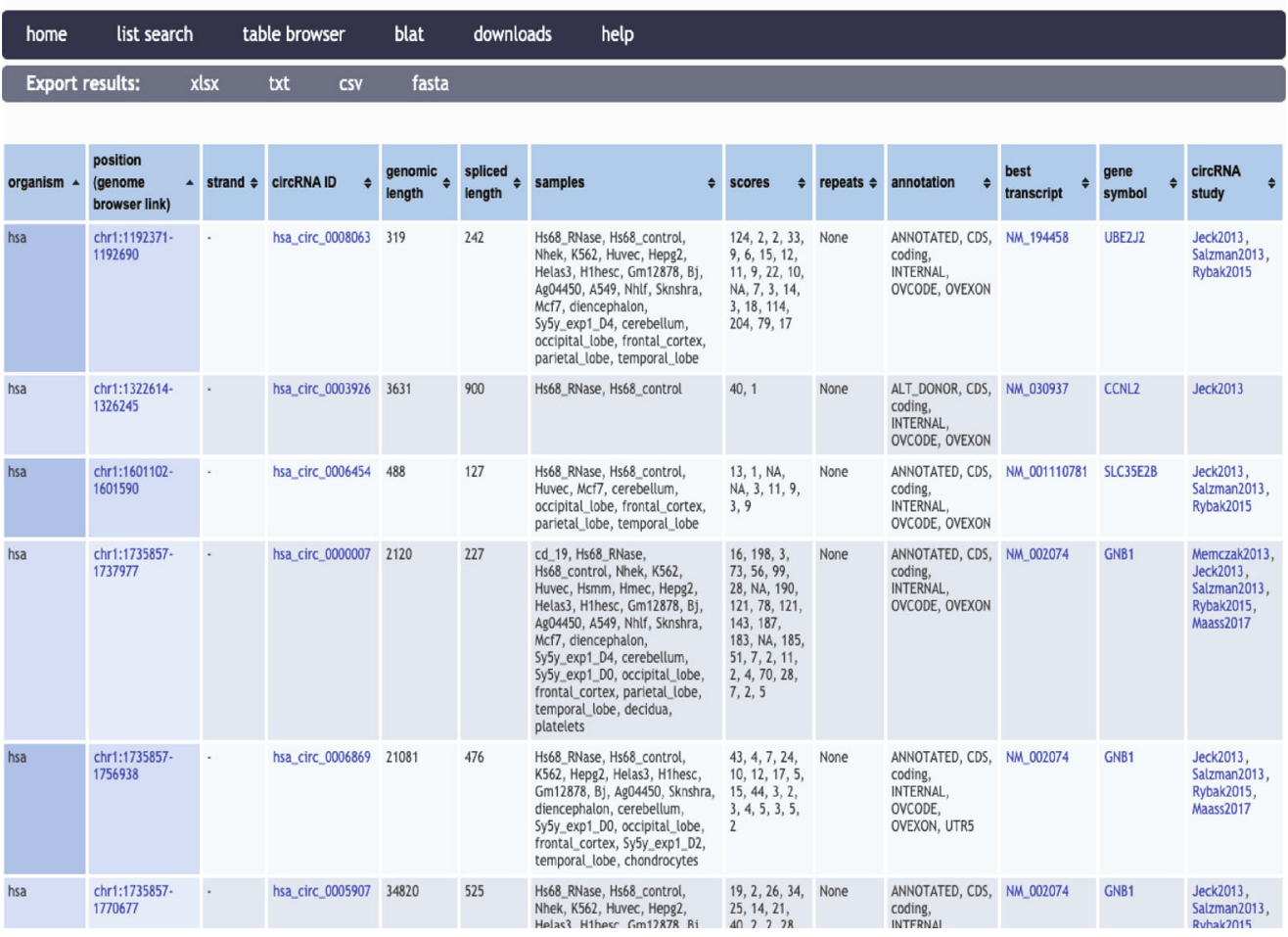

(b)

FIGURE 5: Searching circRNAs with circBase table browser. This illustration gives a brief introduction on how to search circBase using the table browser option. (a) circBase table browser interface. (b) An output from the result page after submitting queries. 
(6) CIRCpedia, a 2nd version of this database, is based on CIRCpedia, for comprehensive circRNA annotation from > 180 RNA-seq datasets [149]; this database contains circRNA annotations across 6 species, including human, mouse, rat, zebrafish, fly, and worm

(7) CircR2Disease is a manually curated database that gives users a comprehensive resource for circRNA deregulation in diseases [150]; it contains $>700$ associations between 661 circRNAs and 100 diseases so that users can study the mechanism of disease-related circRNAs

(8) exoRBase is a database that has $>58 \mathrm{k}$ circRNAs in human blood exosomes, which helps users to identify exosomal biomarkers [151]

(9) TRCirc can be used to study transcriptional regulation of circRNAs based on ChIP-seq and RNAseq results [152]; it also enables analysis of methylation level

(10) CircRNAdisease is another newly developed database to understand circRNA and disease associations [153]; it contains 354 associations between 330 circRNAs and 48 diseases

(11) CircBank is a comprehensive database for human circRNAs, and it contains 5 features such as a miRNA binding site, conservation of circRNAs, m6A modification, mutation, and protein-coding potential of circRNAs [154]; note that this database has a novel naming system for circRNAs

(12) circFunbase is a database featured by a highquality functional circRNA resource [155]; most of the resource has been validated by experiments; it contains circRNAs from a wide variety of species, such as plants and animals (human, monkey, rat, mouse, etc.)

(13) $L n c A C T d b$ is a database mainly for endogenous RNAs such as circRNAs in different species and diseases [156]; it contains about 60 experimentally supported circRNA interactions

(14) $\operatorname{Crop} C \operatorname{ircDB}$ is a database specifically for crop circRNAs such as maize and rice [157]; it also has validated crop circRNAs in response to abiotic stress

(15) AtCircDB is another plant-specific database mainly for Arabidopsis circRNAs [158]

(16) MiOncoCirc is a database that contains circRNAs from cancer cell lines and tumor samples [23]

(17) Circad is another disease-associated database for circRNAs [144]; it has $>1300$ circRNAs implicated with 150 diseases; besides, it has circRNAs from 5 species, including human, rat, and mouse

(18) ncrpheno is a database mainly for ncRNAs; however, it contains 848 circRNAs as well as circRNArelated diseases [159]
(19) NPInter (v4) is the 4th version of the NPInter database that integrates $6 \mathrm{M}$ newly identified ncRNA interactions including circRNA interactions [160]; it also contains circRNAs from dozens of species, including human, mouse and rat

(20) CircAtlas (v2) is a database that integrated $1 \mathrm{M}$ circRNAs across 6 species, including human, macaca, mouse, rat, pit, and chicken as well as 19 normal different tissues [161]; it also describes a conservation score, coexpression, and regulatory networks

(21) VirusCircBase is a comprehensive database of viral circRNAs [162]; it contains $12 \mathrm{~K}$ circRNAs, most in viruses and infectious diseases

To our knowledge, this summary list is the most updated summary of circRNA databases. Here, we recommend the following principles for readers to choose each database based on the purposes of their experiment and analysis:

(1) Disease association: for projects that are aimed at comparing several disease conditions, these databases could be chosen-circ2Traits, circR2Disease, circRNAdisease, Circad, ncrpheno, and CircAtlas

(2) Cross-species comparison: for projects involving a cross-species comparison, these databases contain circRNA information on several different species, including CIRCpedia, circFunbase, Circad, NPInter (v4), and CircAtlas (v2)

(3) Transcriptomic regulation: for projects that are aimed at studying epigenetic regulation of gene expression, these databases could be chosen-TRCirc, CircBank, LncACTdb, and NPInter (v4)

(4) Tissue-specific purpose: for projects that are aimed at comparing circRNAs in a wide range of normal tissues. The databases that could fulfill this purpose are TSCD, NPInter (v4), and CircAtlas (v2). Nevertheless, it is advised to perform an initial search via exoRBase for a blood-related project, whereas VirusCircBase should be the first choice for a virus-related project. However, it is always preferable to go through each relevant database if necessary.

\section{Concluding Remarks}

In the past few years, growing evidence has been seen in circRNAs as potential diagnostic and prognostic biomarkers for human diseases. Because most circRNAs are abundantly expressed in a wide variety of tissue types and cell types, and that circRNAs show great stability and a robust regulation role in gene expression, circRNAs will become favorable biomarker candidates that are worthy of investigation in both basic and clinical medical sciences.

One of the bottlenecks in studying circRNAs is detection and identification from genome-wide datasets. The emerging field of big data enables us an unprecedented opportunity to store, manage, process, and analyze biological data 
that contains information with tremendous complexity. Therefore, a computational strategy that mainly uses publicly available pipelines and databases developed and shared by circRNA communities could enormously reduce the challenges and increase the efficiency of applying bioinformatics knowledge to identify key circRNAs that could bring diagnostic and prognostic values.

In this review, we have briefly introduced the biology of circRNAs, including characteristics, biogenesis, biological functions, and disease relevance, as well as several computational approaches that enable researchers to detect and identify potential novel circRNAs. Finally, we have highlighted several publicly available computational resources for the analysis of circRNAs that, to our knowledge, are the most completed and updated. Thus, we hope this review will help researchers at various levels in their current and future studies on circRNAs.

The study of circRNAs has just begun, and the field is relatively young. A number of outstanding questions are still waiting to be addressed, such as the association of circRNAs in disease progression and development, the value of circulating circRNAs to predict their abundance and relevance in deep tissues, novel functions of circRNAs beyond sponges for small molecules, and the efficiency of combining singlemolecule HTS technology with circRNAs [34, 51, 119, 163]. Nevertheless, the continuous efforts in detection, identification, and characterization of circRNAs will lead to our understanding of circRNAs' function and clinical value into a completely new lever.

\section{Abbreviations}

$\begin{array}{ll}\text { AGO: } & \text { Argonaute protein } \\ \text { APP: } & \beta \text {-Amyloid precursor protein } \\ \text { BACE: } & \beta \text {-Site APP-cleaving enzyme } \\ \text { circRNAs: } & \text { Circular RNAs } \\ \text { ecircRNAs: } & \text { Exon-derived circRNAs } \\ \text { elciRNAs: } & \text { Exon-intron circRNAs } \\ \text { endo-siRNA: } & \text { Endogenous small interfering RNA } \\ \text { HTS: } & \text { High-throughput sequencing } \\ \text { lncRNA: } & \text { Long noncoding RNA } \\ \text { lncRNA: } & \text { Long noncoding RNA } \\ \text { ncRNA: } & \text { Noncoding RNA } \\ \text { pre-mRNA: } & \text { Precursor message RNAs } \\ \text { RBP: } & \text { RNA-binding protein } \\ \text { RNA-seq: } & \text { RNA sequencing } \\ \text { rRNA: } & \text { Ribosomal RNA } \\ \text { sncRNA: } & \text { Small noncoding RNA } \\ \text { tRNA: } & \text { Transfer RNA. }\end{array}$

\section{Disclosure}

The work described here has not been published before, and it is not under consideration for publication anywhere else. Its publication has been approved by all coauthors, as well as by the responsible authorities, if any-tacitly or explicitly-at the institute where the work has been carried out. The publisher will not be held legally responsible should there be any claims for compensation.

\section{Conflicts of Interest}

The authors declare that they have no conflicts of interest.

\section{Acknowledgments}

We thank all the individuals who have helped us in this study. We acknowledge the valuable work of the many investigators whose published articles we were unable to cite owing to space limitations. This work was supported by a grant from Jiangsu University (19JDG039).

\section{References}

[1] J. Salzman, C. Gawad, P. L. Wang, N. Lacayo, and P. O. Brown, "Circular RNAs are the predominant transcript isoform from hundreds of human genes in diverse cell types," PLoS One, vol. 7, no. 2, article e30733, 2012.

[2] J. T. Granados-Riveron and G. Aquino-Jarquin, "The complexity of the translation ability of circRNAs," Biochimica et Biophysica Acta (BBA) - Gene Regulatory Mechanisms, vol. 1859, no. 10, pp. 1245-1251, 2016.

[3] Q. Zheng, C. Bao, W. Guo et al., "Circular RNA profiling reveals an abundant circHIPK3 that regulates cell growth by sponging multiple miRNAs," Nature Communications, vol. 7, no. 1, article 11215, 2016.

[4] Z. Li, C. Huang, C. Bao et al., "Exon-intron circular RNAs regulate transcription in the nucleus," Nature Structural \& Molecular Biology, vol. 22, no. 3, pp. 256-264, 2015.

[5] S. J. Conn, K. A. Pillman, J. Toubia et al., "The RNA binding protein quaking regulates formation of circRNAs," Cell, vol. 160, no. 6, pp. 1125-1134, 2015.

[6] L. V. Stagsted, K. M. Nielsen, I. Daugaard, and T. B. Hansen, "Noncoding AUG circRNAs constitute an abundant and conserved subclass of circles," Life Science Alliance, vol. 2, no. 3, 2019.

[7] L. S. Kristensen, M. S. Andersen, L. V. W. Stagsted, K. K. Ebbesen, T. B. Hansen, and J. Kjems, "The biogenesis, biology and characterization of circular RNAs," Nature Reviews. Genetics, vol. 20, no. 11, pp. 675-691, 2019.

[8] K. Y. Hsiao, Y. C. Lin, S. K. Gupta et al., "Noncoding effects of circular RNA CCDC66 promote colon cancer growth and metastasis," Cancer Research, vol. 77, no. 9, pp. 2339-2350, 2017.

[9] W. Gao, Y. B. Sun, W. W. Zhou et al., "Genomic and transcriptomic investigations of the evolutionary transition from oviparity to viviparity," Proceedings of the National Academy of Sciences of the United States of America, vol. 116, no. 9, pp. 3646-3655, 2019.

[10] D. Hanniford, A. Ulloa-Morales, A. Karz et al., "Epigenetic Silencing of _CDR1as_ Drives IGF2BP3-Mediated Melanoma Invasion and Metastasis," Cancer Cell, vol. 37, no. 1, pp. 55-70.e15, 2020.

[11] F. Zhang, R. Zhang, X. Zhang et al., "Comprehensive analysis of circRNA expression pattern and circRNA-miRNA-mRNA network in the pathogenesis of atherosclerosis in rabbits," Aging, vol. 10, no. 9, pp. 2266-2283, 2018.

[12] M. A. Khan, Y. J. Reckman, S. Aufiero et al., "RBM20 regulates circular RNA production from the Titin gene," Circulation Research, vol. 119, no. 9, pp. 996-1003, 2016. 
[13] L. M. Holdt, A. Stahringer, K. Sass et al., "Circular noncoding RNA ANRIL modulates ribosomal RNA maturation and atherosclerosis in humans," Nature Communications, vol. 7, no. 1, article 12429, 2016.

[14] Y. Fang, X. Wang, W. Li et al., "Screening of circular RNAs and validation of circANKRD36 associated with inflammation in patients with type 2 diabetes mellitus," International Journal of Molecular Medicine, vol. 42, no. 4, pp. 18651874, 2018.

[15] C. Bai, W. Yang, Y. Lu, W. Wei, Z. Li, and L. Zhang, "Identification of Circular RNAs Regulating Islet $\beta$-Cell Autophagy in Type 2 Diabetes Mellitus," BioMed Research International, vol. 2019, Article ID 4128315, 10 pages, 2019.

[16] Z. Shi, T. Chen, Q. Yao et al., "The circularRNAciRS-7 promotesAPPandBACE1 degradation in anNF- $\kappa$ B-dependent manner," The FEBS Journal, vol. 284, no. 7, pp. 1096-1109, 2017.

[17] Y. Zhang, F. Yu, S. Bao, and J. Sun, "Systematic characterization of circular RNA-associated CeRNA network identified novel circRNA biomarkers in Alzheimer's disease," Frontiers in Bioengineering and Biotechnology, vol. 7, p. 222, 2019.

[18] Y. Li, H. Fan, J. Sun et al., "Circular RNA expression profile of Alzheimer's disease and its clinical significance as biomarkers for the disease risk and progression," The International Journal of Biochemistry \& Cell Biology, vol. 123, p. 105747, 2020.

[19] S. Jahani, E. Nazeri, K. Majidzadeh-A, M. Jahani, and R. Esmaeili, "Circular RNA; a new biomarker for breast cancer: a systematic review," Journal of Cellular Physiology, vol. 235, no. 7-8, pp. 5501-5510, 2020.

[20] D. Sekar, "Circular RNA: a new biomarker for different types of hypertension," Hypertension Research, vol. 42, no. 11, pp. 1824-1825, 2019.

[21] B. Lei, Z. Tian, W. Fan, and B. Ni, "Circular RNA: a novel biomarker and therapeutic target for human cancers," International Journal of Medical Sciences, vol. 16, no. 2, pp. 292301, 2019.

[22] B. Chen and S. Huang, "Circular RNA: an emerging noncoding RNA as a regulator and biomarker in cancer," Cancer Letters, vol. 418, pp. 41-50, 2018.

[23] J. N. Vo, M. Cieslik, Y. Zhang et al., "The landscape of circular RNA in cancer," Cell, vol. 176, no. 4, pp. 869-881.e13, 2019.

[24] R. Kishore, V. N. S. Garikipati, and C. Gonzalez, "Role of circular RNAs in cardiovascular disease," Journal of Cardiovascular Pharmacology, vol. 76, no. 2, pp. 128-137, 2020.

[25] S. Aufiero, Y. J. Reckman, Y. M. Pinto, and E. E. Creemers, "Circular RNAs open a new chapter in cardiovascular biology," Nature Reviews Cardiology, vol. 16, no. 8, pp. 503$514,2019$.

[26] K. Abbaszadeh-Goudarzi, S. Radbakhsh, M. H. Pourhanifeh et al., "Circular RNA and diabetes: epigenetic regulator with diagnostic role," Current Molecular Medicine, vol. 20, no. 7, pp. 516-526, 2020.

[27] R. Akhter, "Circular RNA and Alzheimer's disease," Advances in Experimental Medicine and Biology, vol. 1087, pp. 239-243, 2018.

[28] H. L. Sanger, G. Klotz, D. Riesner, H. J. Gross, and A. K. Kleinschmidt, "Viroids are single-stranded covalently closed circular RNA molecules existing as highly base-paired rodlike structures," Proceedings of the National Academy of Sciences of the United States of America, vol. 73, no. 11, pp. 3852-3856, 1976.
[29] M. T. Hsu and M. Coca-Prados, "Electron microscopic evidence for the circular form of RNA in the cytoplasm of eukaryotic cells," Nature, vol. 280, no. 5720, pp. 339-340, 1979.

[30] C. Cocquerelle, B. Mascrez, D. Hetuin, and B. Bailleul, "Missplicing yields circular RNA molecules," The FASEB Journal, vol. 7, no. 1, pp. 155-160, 1993.

[31] W. R. Jeck, J. A. Sorrentino, K. Wang et al., "Circular RNAs are abundant, conserved, and associated with ALU repeats," RNA, vol. 19, no. 2, pp. 141-157, 2013.

[32] S. Memczak, M. Jens, A. Elefsinioti et al., "Circular RNAs are a large class of animal RNAs with regulatory potency," Nature, vol. 495, no. 7441, pp. 333-338, 2013.

[33] R. Dong, X. K. Ma, L. L. Chen, and L. Yang, "Increased complexity of circRNA expression during species evolution," RNA Biology, vol. 14, no. 8, pp. 1064-1074, 2016.

[34] W. Chen and E. Schuman, "Circular RNAs in brain and other tissues: a functional enigma," Trends in Neurosciences, vol. 39, no. 9, pp. 597-604, 2016.

[35] A. Rybak-Wolf, C. Stottmeister, P. Glazar et al., "Circular RNAs in the mammalian brain are highly abundant, conserved, and dynamically expressed," Molecular Cell, vol. 58, no. 5, pp. 870-885, 2015.

[36] A. Zaghlool, A. Ameur, C. Wu et al., "Expression profiling and in situ screening of circular RNAs in human tissues," Scientific Reports, vol. 8, no. 1, article 16953, 2018.

[37] T. Xu, J. Wu, P. Han, Z. Zhao, and X. Song, "Circular RNA expression profiles and features in human tissues: a study using RNA-seq data," BMC Genomics, vol. 18, Supplement 6, p. 680, 2017.

[38] N. R. Pamudurti, O. Bartok, M. Jens et al., "Translation of circRNAs," Molecular Cell, vol. 66, no. 1, pp. 9-21.e7, 2017.

[39] I. Legnini, G. Di Timoteo, F. Rossi et al., "Circ-ZNF609 is a circular RNA that can be translated and functions in myogenesis,” Molecular Cell, vol. 66, no. 1, pp. 22-37.e9, 2017.

[40] D. C. Tatomer and J. E. Wilusz, "An unchartered journey for ribosomes: circumnavigating circular RNAs to produce proteins," Molecular Cell, vol. 66, no. 1, pp. 1-2, 2017.

[41] Y. Yang, X. Fan, M. Mao et al., "Extensive translation of circular RNAs driven by $N^{6}$-methyladenosine," Cell Research, vol. 27, no. 5, pp. 626-641, 2017.

[42] R. A. Wesselhoeft, P. S. Kowalski, and D. G. Anderson, "Engineering circular RNA for potent and stable translation in eukaryotic cells," Nature Communications, vol. 9, no. 1, article 2629, 2018.

[43] E. Lasda and R. Parker, "Circular RNAs: diversity of form and function,” RNA, vol. 20, no. 12, pp. 1829-1842, 2014.

[44] X. O. Zhang, H. B. Wang, Y. Zhang, X. Lu, L. L. Chen, and L. Yang, "Complementary sequence-mediated exon circularization,” Cell, vol. 159, no. 1, pp. 134-147, 2014.

[45] L. Szabo, R. Morey, N. J. Palpant et al., "Statistically based splicing detection reveals neural enrichment and tissuespecific induction of circular RNA during human fetal development," Genome Biology, vol. 16, no. 1, p. 126, 2015.

[46] A. Ivanov, S. Memczak, E. Wyler et al., "Analysis of intron sequences reveals hallmarks of circular RNA biogenesis in animals," Cell Reports, vol. 10, no. 2, pp. 170-177, 2015.

[47] D. Liang and J. E. Wilusz, "Short intronic repeat sequences facilitate circular RNA production," Genes \& Development, vol. 28, no. 20, pp. 2233-2247, 2014. 
[48] L. L. Chen and L. Yang, "Regulation of circRNA biogenesis," RNA Biology, vol. 12, no. 4, pp. 381-388, 2015.

[49] L. L. Chen, "The biogenesis and emerging roles of circular RNAs," Nature Reviews Molecular Cell Biology, vol. 17, no. 4, pp. 205-211, 2016.

[50] X. O. Zhang, R. Dong, Y. Zhang et al., "Diverse alternative back-splicing and alternative splicing landscape of circular RNAs,” Genome Research, vol. 26, no. 9, pp. 1277-1287, 2016.

[51] Z. Zhang, T. Yang, and J. Xiao, "Circular RNAs: promising biomarkers for human diseases," eBioMedicine, vol. 34, pp. 267-274, 2018.

[52] D. W. Thomson and M. E. Dinger, "Endogenous microRNA sponges: evidence and controversy," Nature Reviews Genetics, vol. 17, no. 5, pp. 272-283, 2016.

[53] S. HafezQorani, A. Houdjedj, M. Arici, A. Said, and H. Kazan, "RBPSponge: genome-wide identification of lncRNAs that sponge RBPs," Bioinformatics, vol. 35, no. 22, pp. 47604763, 2019.

[54] L. P. Zhu, Y. J. He, J. C. Hou et al., "The role of circRNAs in cancers," Bioscience Reports, vol. 37, no. 5, 2017.

[55] Q. Tang and S. S. Hann, "Biological roles and mechanisms of circular RNA in human cancers," Oncotargets and Therapy, vol. Volume 13, pp. 2067-2092, 2020.

[56] J. Zang, D. Lu, and A. Xu, "The interaction of circRNAs and RNA binding proteins: an important part of circRNA maintenance and function," Journal of Neuroscience Research, vol. 98, no. 1, pp. 87-97, 2020.

[57] T. B. Hansen, T. I. Jensen, B. H. Clausen et al., "Natural RNA circles function as efficient microRNA sponges," Nature, vol. 495, no. 7441, pp. 384-388, 2013.

[58] W. Weng, Q. Wei, S. Toden et al., "Circular RNA ciRS-7-a promising prognostic biomarker and a potential therapeutic target in colorectal cancer," Clinical Cancer Research, vol. 23, no. 14, pp. 3918-3928, 2017.

[59] L. Xu, M. Zhang, X. Zheng, P. Yi, C. Lan, and M. Xu, “The circular RNA ciRS-7 (Cdrlas) acts as a risk factor of hepatic microvascular invasion in hepatocellular carcinoma," Journal of Cancer Research and Clinical Oncology, vol. 143, no. 1, pp. 17-27, 2017.

[60] R. C. Li, S. Ke, F. K. Meng et al., "CiRS-7 promotes growth and metastasis of esophageal squamous cell carcinoma via regulation of miR-7/HOXB13," Cell Death \& Disease, vol. 9, no. 8 , p. 838, 2018.

[61] H. Huang, L. Wei, T. Qin, N. Yang, Z. Li, and Z. Xu, "Circular RNA ciRS-7 triggers the migration and invasion of esophageal squamous cell carcinoma via miR-7/KLF4 and NF- $\kappa \mathrm{B}$ signals," Cancer Biology \& Therapy, vol. 20, no. 1, pp. 7380, 2018.

[62] C. Su, Y. Han, H. Zhang et al., "CiRS-7 targeting miR-7 modulates the progression of non-small cell lung cancer in a manner dependent on NF- $\kappa$ B signalling," Journal of Cellular and Molecular Medicine, vol. 22, no. 6, pp. 3097-3107, 2018.

[63] L. Liu, F. B. Liu, M. Huang et al., "Circular RNA ciRS-7 promotes the proliferation and metastasis of pancreatic cancer by regulating miR-7-mediated EGFR/STAT3 signaling pathway," Hepatobiliary \& Pancreatic Diseases International, vol. 18, no. 6, pp. 580-586, 2019.

[64] M. Piwecka, P. Glazar, L. R. Hernandez-Miranda et al., "Loss of a mammalian circular RNA locus causes miRNA deregulation and affects brain function," Science, vol. 357, no. 6357, article eaam8526, 2017.
[65] H. Gruner, M. Cortes-Lopez, D. A. Cooper, M. Bauer, and P. Miura, "circRNA accumulation in the aging mouse brain," Scientific Reports, vol. 6, no. 1, article 38907, 2016.

[66] C. Lu, X. Sun, N. Li et al., "circRNAs in the tree shrew (Tupaia belangeri) brain during postnatal development and aging," Aging, vol. 10, no. 4, pp. 833-852, 2018.

[67] K. Xu, D. Chen, Z. Wang et al., "Annotation and functional clustering of circRNA expression in rhesus macaque brain during aging," Cell Discovery, vol. 4, no. 1, p. 48, 2018.

[68] A. S. Reddy, D. O’Brien, N. Pisat et al., "A comprehensive analysis of cell type-specific nuclear RNA from neurons and glia of the brain," Biological Psychiatry, vol. 81, no. 3, pp. 252-264, 2017.

[69] B. Kleaveland, C. Y. Shi, J. Stefano, and D. P. Bartel, "A network of noncoding regulatory RNAs acts in the mammalian brain," Cell, vol. 174, no. 2, pp. 350-362.e17, 2018.

[70] I. B. Filippenkov, T. A. Kolomin, S. A. Limborska, and L. V. Dergunova, "Developmental stage-specific expression of genes for sphingomyelin synthase in rat brain," Cell and Tissue Research, vol. 372, no. 1, pp. 33-40, 2018.

[71] F. Xie, Y. Zhao, S. D. Wang, J. Ma, X. Wang, and L. J. Qian, "Identification, characterization, and functional investigation of circular RNAs in subventricular zone of adult rat brain," Journal of Cellular Biochemistry, vol. 120, no. 3, pp. 34283437, 2018.

[72] C. Suenkel, D. Cavalli, S. Massalini, F. Calegari, and N. Rajewsky, "A highly conserved circular RNA is required to keep neural cells in a progenitor state in the mammalian brain," Cell Reports, vol. 30, no. 7, pp. 2170-2179.e5, 2020.

[73] B. J. Chen, S. Huang, and M. Janitz, "Changes in circular RNA expression patterns during human foetal brain development," Genomics, vol. 111, no. 4, pp. 753-758, 2019.

[74] B. J. Chen, J. D. Mills, K. Takenaka, N. Bliim, G. M. Halliday, and M. Janitz, "Characterization of circular RNAs landscape in multiple system atrophy brain," Journal of Neurochemistry, vol. 139, no. 3, pp. 485-496, 2016.

[75] S. L. Mehta, G. Pandi, and R. Vemuganti, "Circular RNA expression profiles alter significantly in mouse brain after transient focal ischemia," Stroke, vol. 48, no. 9, pp. 25412548, 2017.

[76] Y. Bai, Y. Zhang, B. Han et al., "Circular RNA DLGAP4 ameliorates ischemic stroke outcomes by targeting miR-143 to regulate endothelial-mesenchymal transition associated with blood-brain barrier integrity," The Journal of Neuroscience, vol. 38, no. 1, pp. 32-50, 2018.

[77] B. S. Xie, Y. Q. Wang, Y. Lin et al., "Circular RNA expression profiles alter significantly after traumatic brain injury in rats," Journal of Neurotrauma, vol. 35, no. 14, pp. 16591666, 2018.

[78] R. T. Zhao, J. Zhou, X. L. Dong et al., "Circular ribonucleic acid expression alteration in exosomes from the brain extracellular space after traumatic brain injury in mice," Journal of Neurotrauma, vol. 35, no. 17, pp. 2056-2066, 2018.

[79] Z. Chen, H. Wang, J. Zhong et al., "Significant changes in circular RNA in the mouse cerebral cortex around an injury site after traumatic brain injury," Experimental Neurology, vol. 313, pp. 37-48, 2019.

[80] L. Jiang, H. Li, Z. Fan, R. Zhao, and Z. Xia, "Circular RNA expression profiles in neonatal rats following hypoxicischemic brain damage," International Journal of Molecular Medicine, vol. 43, no. 4, pp. 1699-1708, 2019. 
[81] Y. J. Jiang, S. Q. Cao, L. B. Gao et al., "Circular ribonucleic acid expression profile in mouse cortex after traumatic brain injury," Journal of Neurotrauma, vol. 36, no. 7, pp. 10181028, 2019.

[82] W. Liu, C. Jia, L. Luo et al., "Novel circular RNAs expressed in brain microvascular endothelial cells after oxygen-glucose deprivation/recovery," Neural Regeneration Research, vol. 14, no. 12, pp. 2104-2111, 2019.

[83] S. Zhang, D. Zhu, H. Li, H. Li, C. Feng, and W. Zhang, "Characterization of circRNA-Associated-ceRNA Networks in a Senescence- Accelerated Mouse Prone 8 Brain," Molecular Therapy, vol. 25, no. 9, pp. 2053-2061, 2017.

[84] N. Ma, J. Pan, X. Ye, B. Yu, W. Zhang, and J. Wan, "Wholetranscriptome analysis of APP/PS1 mouse brain and identification of circRNA-miRNA-mRNA networks to investigate AD pathogenesis," Molecular Therapy-Nucleic Acids, vol. 18, pp. 1049-1062, 2019.

[85] E. Jia, Y. Zhou, Z. Liu et al., "Transcriptomic profiling of circular RNA in different brain regions of Parkinson's disease in a mouse model," International Journal of Molecular Sciences, vol. 21, no. 8, p. 3006, 2020.

[86] R. Yang, B. Xu, B. Yang et al., "Circular RNA transcriptomic analysis of primary human brain microvascular endothelial cells infected with meningitic Escherichia coli," Molecular Therapy-Nucleic Acids, vol. 13, pp. 651-664, 2018.

[87] K. Xu, L. Ding, T. C. Chang et al., "Structure and evolution of double minutes in diagnosis and relapse brain tumors," Acta Neuropathologica, vol. 137, no. 1, pp. 123-137, 2019.

[88] G. Yoon, K. A. Cho, J. Song, and Y. K. Kim, “Transcriptomic analysis of high fat diet fed mouse brain cortex," Frontiers in Genetics, vol. 10, p. 83, 2019.

[89] W. L. Tan, B. T. Lim, C. G. Anene-Nzelu et al., "A landscape of circular RNA expression in the human heart," Cardiovascular Research, vol. 113, no. 3, pp. 298-309, 2017.

[90] K. Wang, B. Long, F. Liu et al., "A circular RNA protects the heart from pathological hypertrophy and heart failure by targeting miR-223," European Heart Journal, vol. 37, no. 33, pp. 2602-2611, 2016.

[91] H. J. Wu, C. Y. Zhang, S. Zhang, M. Chang, and H. Y. Wang, "Microarray expression profile of circular RNAs in heart tissue of mice with myocardial infarction-induced heart failure," Cellular Physiology and Biochemistry, vol. 39, no. 1, pp. 205-216, 2016.

[92] M. S. Hulshoff, X. Xu, G. Krenning, and E. M. Zeisberg, "Epigenetic regulation of endothelial-to-mesenchymal transition in chronic heart disease," Arteriosclerosis, Thrombosis, and Vascular Biology, vol. 38, no. 9, pp. 1986-1996, 2018.

[93] Y. Yang, H. Chen, N. Ding et al., "Expression profiling of circular RNAs and microRNAs in heart tissue of mice with alcoholic cardiomyopathy," Cellular Physiology and Biochemistry, vol. 46, no. 6, pp. 2284-2296, 2018.

[94] X. Ge, Q. Meng, R. Zhuang et al., "Circular RNA expression alterations in extracellular vesicles isolated from murine heart post ischemia/reperfusion injury," International Journal of Cardiology, vol. 296, pp. 136-140, 2019.

[95] Y. Zhang, G. Zhang, Y. Liu et al., "GDF15 regulates Malat-1 circular RNA and inactivates $\mathrm{NF} \kappa \mathrm{B}$ signaling leading to immune tolerogenic DCs for preventing alloimmune rejection in heart transplantation," Frontiers in Immunology, vol. 9, p. 2407, 2018.
[96] M. Hu, X. Wei, M. Li et al., "Circular RNA expression profiles of persistent atrial fibrillation in patients with rheumatic heart disease," Anatolian Journal of Cardiology, vol. 21, no. 1, pp. 2-10, 2019.

[97] J. Han, L. Zhang, L. Hu et al., "Circular RNA-expression profiling reveals a potential role of Hsa_circ_0097435 in heart failure via sponging multiple microRNAs," Frontiers in Genetics, vol. 11, p. 212, 2020.

[98] Y. Sun, X. Jiang, Y. Lv et al., "Circular RNA expression profiles in plasma from patients with heart failure related to platelet activity," Biomolecules, vol. 10, no. 2, p. 187, 2020.

[99] H. Liu, Y. Hu, B. Zhuang et al., "Differential expression of circRNAs in embryonic heart tissue associated with ventricular septal defect," International Journal of Medical Sciences, vol. 15, no. 7, pp. 703-712, 2018.

[100] F. Lin, G. Zhao, Z. Chen et al., "circRNA-miRNA association for coronary heart disease," Molecular Medicine Reports, vol. 19, no. 4, pp. 2527-2536, 2019.

[101] Y. Sun, R. Chen, S. Lin et al., "Association of circular RNAs and environmental risk factors with coronary heart disease," BMC Cardiovascular Disorders, vol. 19, no. 1, p. 223, 2019.

[102] F. Yu, Y. Tie, Y. Zhang et al., "Circular RNA expression profiles and bioinformatic analysis in coronary heart disease," Epigenomics, vol. 12, no. 5, pp. 439-454, 2020.

[103] A. Bachmayr-Heyda, A. T. Reiner, K. Auer et al., "Correlation of circular RNA abundance with proliferation - exemplified with colorectal and ovarian cancer, idiopathic lung fibrosis and normal human tissues," Scientific Reports, vol. 5, no. 1, article 8057, 2015.

[104] X. Dai, N. Zhang, Y. Cheng et al., "RNA-binding protein trinucleotide repeat-containing $6 \mathrm{~A}$ regulates the formation of circular RNA circ0006916, with important functions in lung cancer cells," Carcinogenesis, vol. 39, no. 8, pp. 981-992, 2018.

[105] D. Hang, J. Zhou, N. Qin et al., “A novel plasma circular RNA circFARSA is a potential biomarker for non-small cell lung cancer," Cancer Medicine, vol. 7, no. 6, pp. 2783-2791, 2018.

[106] X. Li, Z. Zhang, H. Jiang et al., "Circular RNA circPVT1 promotes proliferation and invasion through sponging miR$125 \mathrm{~b}$ and activating E2F2 signaling in non-small cell lung cancer," Cellular Physiology and Biochemistry, vol. 51, no. 5, pp. 2324-2340, 2018.

[107] X. Ma, X. Yang, W. Bao et al., "Circular RNA circMAN2B2 facilitates lung cancer cell proliferation and invasion via miR-1275/FOXK1 axis," Biochemical and Biophysical Research Communications, vol. 498, no. 4, pp. 1009-1015, 2018.

[108] S. Tan, D. Sun, W. Pu et al., "Circular RNA F-circEA-2a derived from EML4-ALK fusion gene promotes cell migration and invasion in non-small cell lung cancer," Molecular Cancer, vol. 17, no. 1, p. 138, 2018.

[109] M. M. Jiang, Z. T. Mai, S. Z. Wan et al., "Microarray profiles reveal that circular RNA hsa_circ_0007385 functions as an oncogene in non-small cell lung cancer tumorigenesis," Journal of Cancer Research and Clinical Oncology, vol. 144, no. 4, pp. 667-674, 2018.

[110] F. Tian, C. T. Yu, W. D. Ye, and Q. Wang, "Cinnamaldehyde induces cell apoptosis mediated by a novel circular RNA hsa circ_0043256 in non-small cell lung cancer," Biochemical and Biophysical Research Communications, vol. 493, no. 3, pp. 1260-1266, 2017. 
[111] Y. H. Luo, X. Z. Zhu, K. W. Huang et al., "Emerging roles of circular RNA hsa_circ_0000064 in the proliferation and metastasis of lung cancer," Biomedicine \& Pharmacotherapy, vol. 96, pp. 892-898, 2017.

[112] X. Zhu, X. Wang, S. Wei et al., "hsa_circ_0013958: a circular RNA and potential novel biomarker for lung adenocarcinoma," The FEBS Journal, vol. 284, no. 14, pp. 2170-2182, 2017.

[113] X. Li, Z. Yuan, J. Chen et al., "Microarray analysis reveals the changes of circular RNA expression and molecular mechanism in acute lung injury mouse model," Journal of Cellular Biochemistry, vol. 120, no. 10, pp. 16658-16667, 2019.

[114] J. Wang, M. C. Zhu, B. Kalionis et al., "Characteristics of circular RNA expression in lung tissues from mice with hypoxia-induced pulmonary hypertension," International Journal of Molecular Medicine, vol. 42, no. 3, pp. 13531366, 2018.

[115] P. R. Pandey, R. Munk, G. Kundu, S. De, K. Abdelmohsen, and M. Gorospe, "Methods for analysis of circular RNAs," WIREs RNA, vol. 11, no. 1, article e1566, 2020.

[116] M. Vromman, J. Vandesompele, and P. J. Volders, "Closing the circle: current state and perspectives of circular RNA databases," Briefings in Bioinformatics, 2020.

[117] W. Tang, K. Fu, H. Sun, D. Rong, H. Wang, and H. Cao, “circRNA microarray profiling identifies a novel circulating biomarker for detection of gastric cancer," Molecular Cancer, vol. 17, no. 1, p. 137, 2018.

[118] S. Qu, W. Song, X. Yang et al., "Microarray expression profile of circular RNAs in human pancreatic ductal adenocarcinoma," Genom Data, vol. 5, pp. 385-387, 2015.

[119] W. R. Jeck and N. E. Sharpless, "Detecting and characterizing circular RNAs," Nature Biotechnology, vol. 32, no. 5, pp. 453461, 2014.

[120] X. Zeng, W. Lin, M. Guo, and Q. Zou, "A comprehensive overview and evaluation of circular RNA detection tools," PLoS Computational Biology, vol. 13, no. 6, article e1005420, 2017.

[121] X. K. Ma, M. R. Wang, C. X. Liu et al., "CIRCexplorer3: a CLEAR pipeline for direct comparison of circular and linear RNA expression," Genomics, Proteomics \& Bioinformatics, vol. 17, no. 5, pp. 511-521, 2019.

[122] J. U. Guo, V. Agarwal, H. Guo, and D. P. Bartel, "Expanded identification and characterization of mammalian circular RNAs," Genome Biology, vol. 15, no. 7, p. 409, 2014.

[123] S. Sekar, P. Geiger, L. Cuyugan et al., "Identification of circular RNAs using RNA sequencing," Journal of Visualized Experiments, no. 153, 2019.

[124] X. Meng, X. Li, P. Zhang, J. Wang, Y. Zhou, and M. Chen, "Circular RNA: an emerging key player in RNA world," Briefings in Bioinformatics, vol. 18, no. 4, pp. 547-557, 2016.

[125] T. J. Chuang, C. S. Wu, C. Y. Chen, L. Y. Hung, T. W. Chiang, and M. Y. Yang, "NCLscan: accurate identification of non-colinear transcripts (fusion, trans-splicing and circular RNA) with a good balance between sensitivity and precision," Nucleic Acids Research, vol. 44, no. 3, article e29, 2016.

[126] O. G. Izuogu, A. A. Alhasan, H. M. Alafghani, M. SantibanezKoref, D. J. Elliott, and M. S. Jackson, "PTESFinder: a computational method to identify post-transcriptional exon shuffling (PTES) events," BMC Bioinformatics, vol. 17, no. 1, p. $31,2016$.
[127] L. Szabo and J. Salzman, "Detecting circular RNAs: bioinformatic and experimental challenges," Nature Reviews Genetics, vol. 17, no. 11, pp. 679-692, 2016.

[128] T. B. Hansen, M. T. Veno, C. K. Damgaard, and J. Kjems, "Comparison of circular RNA prediction tools," Nucleic Acids Research, vol. 44, no. 6, article e58, 2016.

[129] Y. Shen, X. Guo, and W. Wang, "Identification and characterization of circular RNAs in zebrafish," FEBS Letters, vol. 591, no. 1, pp. 213-220, 2017.

[130] M. Carrara, P. Fuschi, C. Ivan, and F. Martelli, "Circular RNAs: methodological challenges and perspectives in cardiovascular diseases," Journal of Cellular and Molecular Medicine, vol. 22, no. 11, pp. 5176-5187, 2018.

[131] S. Ghosal, S. Das, R. Sen, P. Basak, and J. Chakrabarti, "Circ2Traits: a comprehensive database for circular RNA potentially associated with disease and traits," Frontiers in Genetics, vol. 4, p. 283, 2013.

[132] P. Glazar, P. Papavasileiou, and N. Rajewsky, "circBase: a database for circular RNAs," RNA, vol. 20, no. 11, pp. 1666-1670, 2014.

[133] S. H. Aghaee-Bakhtiari, "Online databases and circular RNAs," Advances in Experimental Medicine and Biology, vol. 1087, pp. 35-38, 2018.

[134] P. Li, S. Chen, H. Chen et al., "Using circular RNA as a novel type of biomarker in the screening of gastric cancer," Clinica Chimica Acta, vol. 444, pp. 132-136, 2015.

[135] G. Tang, W. Xie, C. Qin et al., "Expression of circular RNA circASXL1 correlates with TNM classification and predicts overall survival in bladder cancer," International Journal of Clinical and Experimental Pathology, vol. 10, no. 8, pp. 8495-8502, 2017.

[136] W. Jia, B. Xu, and J. Wu, "Circular RNA expression profiles of mouse ovaries during postnatal development and the function of circular RNA epidermal growth factor receptor in granulosa cells," Metabolism, vol. 85, pp. 192-204, 2018.

[137] M. Li, Y. Liu, X. Zhang, J. Liu, and P. Wang, “Transcriptomic analysis of high-throughput sequencing about circRNA, lncRNA and mRNA in bladder cancer," Gene, vol. 677, pp. 189-197, 2018.

[138] L. Zhang, Y. Li, W. Liu, H. Li, and Z. Zhu, "Analysis of the complex interaction of CDRlas-miRNA-protein and detection of its novel role in melanoma," Oncology Letters, vol. 16, no. 1, pp. 1219-1225, 2018.

[139] Y. Hong, H. Qin, Y. Li et al., "FNDC3B circular RNA promotes the migration and invasion of gastric cancer cells via the regulation of E-cadherin and CD44 expression," Journal of Cellular Physiology, vol. 234, no. 11, pp. 19895-19910, 2019.

[140] X. Dou, L. Feng, N. Ying et al., "RNA sequencing reveals a comprehensive circular RNA expression profile in a mouse model of alcoholic liver disease," Alcoholism, Clinical and Experimental Research, vol. 44, no. 2, pp. 415-422, 2020.

[141] L. Wang, Y. Liang, Q. Mao et al., "Circular RNA circCRIM1 inhibits invasion and metastasis in lung adenocarcinoma through the microRNA (miR)-182/miR-93-leukemia inhibitory factor receptor pathway," Cancer Science, vol. 110, no. 9, pp. 2960-2972, 2019.

[142] J. Yang, Y. Gong, Q. Jiang et al., "Circular RNA expression profiles in nasopharyngeal carcinoma by sequence analysis," Frontiers in Oncology, vol. 10, p. 601, 2020. 
[143] H. Li, B. Heng, P. Ouyang et al., "Comprehensive profiling of circRNAs and the tumor suppressor function of circHIPK 3 in clear cell renal carcinoma," Journal of Molecular Histology, vol. 51, no. 3, pp. 317-327, 2020.

[144] M. Rophina, D. Sharma, M. Poojary, and V. Scaria, "Circad: a comprehensive manually curated resource of circular RNA associated with diseases," Database, vol. 2020, 2020.

[145] S. Hoffmann, C. Otto, G. Doose et al., "A multi-split mapping algorithm for circular RNA, splicing, trans-splicing and fusion detection," Genome Biology, vol. 15, no. 2, article R34, 2014.

[146] Y. C. Liu, J. R. Li, C. H. Sun et al., "CircNet: a database of circular RNAs derived from transcriptome sequencing data," Nucleic Acids Research, vol. 44, no. D1, pp. D209-D215, 2016.

[147] X. Chen, P. Han, T. Zhou, X. Guo, X. Song, and Y. Li, “circRNADb: a comprehensive database for human circular RNAs with protein- coding annotations," Scientific Reports, vol. 6, no. 1, article 34985, 2016.

[148] S. Xia, J. Feng, L. Lei et al., "Comprehensive characterization of tissue-specific circular RNAs in the human and mouse genomes," Briefings in Bioinformatics, vol. 18, no. 6, pp. 984-992, 2017.

[149] R. Dong, X. K. Ma, G. W. Li, and L. Yang, “CIRCpedia v2: an updated database for comprehensive circular RNA annotation and expression comparison," Genomics, Proteomics \& Bioinformatics, vol. 16, no. 4, pp. 226-233, 2018.

[150] C. Fan, X. Lei, Z. Fang, Q. Jiang, and F. X. Wu, "CircR2Disease: a manually curated database for experimentally supported circular RNAs associated with various diseases," Database, vol. 2018, 2018.

[151] S. Li, Y. Li, B. Chen et al., "exoRBase: a database of circRNA, lncRNA and mRNA in human blood exosomes," Nucleic Acids Research, vol. 46, no. D1, pp. D106-D112, 2018.

[152] Z. Tang, X. Li, J. Zhao et al., "TRCirc: a resource for transcriptional regulation information of circRNAs," Briefings in Bioinformatics, vol. 20, no. 6, pp. 2327-2333, 2019.

[153] Z. Zhao, K. Wang, F. Wu et al., "circRNA disease: a manually curated database of experimentally supported circRNAdisease associations," Cell Death \& Disease, vol. 9, no. 5, p. $475,2018$.

[154] M. Liu, Q. Wang, J. Shen, B. B. Yang, and X. Ding, "Circbank: a comprehensive database for circRNA with standard nomenclature," RNA Biology, vol. 16, no. 7, pp. 899-905, 2019.

[155] X. Meng, D. Hu, P. Zhang, Q. Chen, and M. Chen, "CircFunBase: a database for functional circular RNAs," Database, vol. 2019, 2019.

[156] P. Wang, X. Li, Y. Gao et al., "LncACTdb 2.0: an updated database of experimentally supported ceRNA interactions curated from low- and high-throughput experiments," Nucleic Acids Research, vol. 47, no. D1, pp. D121-D127, 2019.

[157] K. Wang, C. Wang, B. Guo et al., "CropCircDB: a comprehensive circular RNA resource for crops in response to abiotic stress," Database, vol. 2019, 2019.

[158] J. Ye, L. Wang, S. Li et al., "AtCircDB: a tissue-specific database for Arabidopsis circular RNAs," Briefings in Bioinformatics, vol. 20, no. 1, pp. 58-65, 2019.

[159] W. Zhang, G. Yao, J. Wang et al., "Epigenomics-based identification of oestrogen-regulated long noncoding RNAs in ER+ breast cancer," RNA Biology, pp. 1-13, 2020.
[160] X. Teng, X. Chen, H. Xue et al., "NPInter v4.0: an integrated database of ncRNA interactions," Nucleic Acids Research, vol. 48, no. D1, pp. D160-D165, 2020.

[161] W. Wu, P. Ji, and F. Zhao, "CircAtlas: an integrated resource of one million highly accurate circular RNAs from 1070 vertebrate transcriptomes," Genome Biology, vol. 21, no. 1, p. 101, 2020.

[162] Z. Cai, Y. Fan, Z. Zhang et al., "VirusCircBase: a database of virus circular RNAs," Briefings in Bioinformatics, 2020.

[163] Y. Gao and F. Zhao, "Computational strategies for exploring circular RNAs," Trends in Genetics, vol. 34, no. 5, pp. 389400, 2018.

[164] K. M. Nielsen and T. B. Hansen, "CircCCDC66: the colorectal oncogene," Non-coding RNA Investigation, vol. 1, no. 2, p. 3, 2017.

[165] K. Wang, D. Singh, Z. Zeng et al., "MapSplice: accurate mapping of RNA-seq reads for splice junction discovery," Nucleic Acids Research, vol. 38, no. 18, article e178, 2010.

[166] Y. Zhang, X. O. Zhang, T. Chen et al., "Circular intronic long noncoding RNAs,” Molecular Cell, vol. 51, no. 6, pp. 792-806, 2013.

[167] J. O. Westholm, P. Miura, S. Olson et al., "Genome-wide Analysis of Drosophila Circular RNAs Reveals Their Structural and Sequence Properties and Age-Dependent Neural Accumulation," Cell Reports, vol. 9, no. 5, pp. 1966-1980, 2014.

[168] Y. Gao, J. Wang, and F. Zhao, "CIRI: an efficient and unbiased algorithm for de novo circular RNA identification," Genome Biology, vol. 16, no. 1, p. 4, 2015.

[169] Y. Gao, J. Zhang, and F. Zhao, "Circular RNA identification based on multiple seed matching," Briefings in Bioinformatics, vol. 19, no. 5, pp. 803-810, 2018.

[170] E. Andres-Leon, R. Nunez-Torres, and A. M. Rojas, "miARma-Seq: a comprehensive tool for miRNA, mRNA and circRNA analysis," Scientific Reports, vol. 6, no. 1, article 25749, 2016

[171] X. Song, N. Zhang, P. Han et al., "Circular RNA profile in gliomas revealed by identification tool UROBORUS," Nucleic Acids Research, vol. 44, no. 9, article e87, 2016.

[172] L. L. Zheng, J. H. Li, J. Wu et al., "deepBase v2.0: identification, expression, evolution and function of small RNAs, LncRNAs and circular RNAs from deep-sequencing data," Nucleic Acids Research, vol. 44, no. D1, pp. D196-D202, 2016.

[173] X. You, I. Vlatkovic, A. Babic et al., "Neural circular RNAs are derived from synaptic genes and regulated by development and plasticity," Nature Neuroscience, vol. 18, no. 4, pp. 603610, 2015.

[174] L. Chen, Y. Yu, X. Zhang, C. Liu, C. Ye, and L. Fan, "PcircRNA_finder: a software for circRNA prediction in plants," Bioinformatics, vol. 32, no. 22, pp. 3528-3529, 2016.

[175] L. Li, Y. C. Zheng, M. U. R. Kayani et al., "Comprehensive analysis of circRNA expression profiles in humans by RAISE," International Journal of Oncology, vol. 51, no. 6, pp. 1625-1638, 2017.

[176] X. Meng, Q. Chen, P. Zhang, and M. Chen, "CircPro: an integrated tool for the identification of circRNAs with proteincoding potential," Bioinformatics, vol. 33, no. 20, pp. 33143316, 2017.

[177] C. Zhou, B. Molinie, K. Daneshvar et al., "Genome-wide maps of m6A circRNAs identify widespread and cell-type- 
specific methylation patterns that are distinct from mRNAs," Cell Reports, vol. 20, no. 9, pp. 2262-2276, 2017.

[178] Y. Gao, H. Wang, H. Zhang, Y. Wang, J. Chen, and L. Gu, "PRAPI: post-transcriptional regulation analysis pipeline for Iso-Seq," Bioinformatics, vol. 34, no. 9, pp. 15801582, 2018.

[179] D. Wang, "hppRNA - a Snakemake-based handy parameterfree pipeline for RNA-Seq analysis of numerous samples," Briefings in Bioinformatics, vol. 19, no. 4, pp. 622-626, 2018.

[180] H. Yu, B. Jiao, L. Lu et al., "NetMiner-an ensemble pipeline for building genome-wide and high-quality gene coexpression network using massive-scale RNA-seq samples," PLoS One, vol. 13, no. 2, article e0192613, 2018.

[181] G. Ferrero, N. Licheri, L. Coscujuela Tarrero et al., "Docker4Circ: a framework for the reproducible characterization of circRNAs from RNA-Seq data," International Journal of Molecular Sciences, vol. 21, no. 1, p. 293, 2020.

[182] T. Jakobi, A. Uvarovskii, and C. Dieterich, "circtools-a onestop software solution for circular RNA research," Bioinformatics, vol. 35, no. 13, pp. 2326-2328, 2019.

[183] G. Y. Jia, D. L. Wang, M. Z. Xue et al., "CircRNAFisher: a systematic computational approach for de novo circular RNA identification," Acta Pharmacologica Sinica, vol. 40, no. 1, pp. 55-63, 2019.

[184] R. Dong, X. K. Ma, L. L. Chen, and L. Yang, "Genome-wide annotation of circRNAs and their alternative back-splicing/splicing with CIRCexplorer pipeline," Methods in Molecular Biology, vol. 1870, pp. 137-149, 2019.

[185] P. Sun and G. Li, "CircCode: a powerful tool for identifying circRNA coding ability," Frontiers in Genetics, vol. 10, p. $981,2019$.

[186] H. Asghari, Y. Y. Lin, Y. Xu, E. Haghshenas, C. C. Collins, and F. Hach, "CircMiner: accurate and rapid detection of circular RNA through splice-aware pseudo-alignment scheme," Bioinformatics, vol. 36, no. 12, pp. 3703-3711, 2020.

[187] M. Chaabane, R. M. Williams, A. T. Stephens, and J. W. Park, "circDeep: deep learning approach for circular RNA classification from other long non-coding RNA," Bioinformatics, vol. 36, no. 1, pp. 73-80, 2020.

[188] X. Li and Y. Wu, "Detecting circular RNA from highthroughput sequence data with de Bruijn graph," BMC Genomics, vol. 21, Suppl 1, p. 749, 2020.

[189] A. Nedoluzhko, F. Sharko, M. G. Rbbani, A. Teslyuk, I. Konstantinidis, and J. M. O. Fernandes, "CircParser: a novel streamlined pipeline for circular RNA structure and host gene prediction in non-model organisms," PeerJ, vol. 8, article e8757, 2020.

[190] J. Cheng, F. Metge, and C. Dieterich, "Specific identification and quantification of circular RNAs from sequencing data," Bioinformatics, vol. 32, no. 7, pp. 1094-1096, 2016.

[191] Y. Gao, J. Wang, Y. Zheng, J. Zhang, S. Chen, and F. Zhao, "Comprehensive identification of internal structure and alternative splicing events in circular RNAs," Nature Communications, vol. 7, no. 1, article 12060, 2016.

[192] M. Li, X. Xie, J. Zhou et al., "Quantifying circular RNA expression from RNA-seq data using model-based framework," Bioinformatics, vol. 33, no. 14, pp. 2131-2139, 2017.

[193] F. Metge, L. F. Czaja-Hasse, R. Reinhardt, and C. Dieterich, "FUCHS-towards full circular RNA characterization using RNAseq," PeerJ, vol. 5, article e2934, 2017.
[194] L. Li, D. Bu, and Y. Zhao, "CircRNAwrap-a flexible pipeline for circRNA identification, transcript prediction, and abundance estimation," FEBS Letters, vol. 593, no. 11, pp. 11791189, 2019.

[195] Y. Zheng, P. Ji, S. Chen, L. Hou, and F. Zhao, "Reconstruction of full-length circular RNAs enables isoform-level quantification," Genome Medicine, vol. 11, no. 1, p. 2, 2019.

[196] L. Chen, F. Wang, E. C. Bruggeman, C. Li, and B. Yao, "circMeta: a unified computational framework for genomic feature annotation and differential expression analysis of circular RNAs," Bioinformatics, vol. 36, no. 2, pp. 539-545, 2020.

[197] J. Zhang, S. Chen, J. Yang, and F. Zhao, “Accurate quantification of circular RNAs identifies extensive circular isoform switching events," Nature Communications, vol. 11, no. 1, p. 90, 2020.

[198] A. Bhattacharya and Y. Cui, "SomamiR 2.0: a database of cancer somatic mutations altering microRNA-ceRNA interactions," Nucleic Acids Research, vol. 44, no. D1, pp. D1005D1010, 2016.

[199] Y. Zou, S. Zheng, X. Deng et al., "The role of circular RNA CDRlas/ciRS-7 in regulating tumor microenvironment: a pan-cancer analysis," Biomolecules, vol. 9, no. 9, p. 429, 2019. 